\title{
ON SYMPLECTIC COVERINGS OF THE PROJECTIVE PLANE
}

\author{
G.-M. GREUEL AND VIK.S. KULIKOV
}

\begin{abstract}
We prove that a resolution of singularities of any finite covering of the projective plane branched along a Hurwitz curve $\bar{H}$ and, maybe, along a line "at infinity" can be embedded as a symplectic submanifold into some projective algebraic manifold equipped with an integer Kähler symplectic form (assuming that if $\bar{H}$ has negative nodes, then the covering is non-singular over them). For cyclic coverings we can realize this embeddings into a rational algebraic 3-fold. Properties of the Alexander polynomial of $\bar{H}$ are investigated and applied to the calculation of the first Betti number $b_{1}\left(\bar{X}_{n}\right)$ of a resolution $\bar{X}_{n}$ of singularities of $n$-sheeted cyclic coverings of $\mathbb{C P}^{2}$ branched along $\bar{H}$ and, maybe, along a line "at infinity". We prove that $b_{1}\left(\bar{X}_{n}\right)$ is even if $\bar{H}$ is an irreducible Hurwitz curve but, in contrast to the algebraic case, that it can take any non-negative value in the case when $\bar{H}$ consists of several irreducible components.
\end{abstract}

\section{INTRODUCTION}

The notion of Hurwitz curves in the projective plane $\mathbb{C P}^{2}$ with respect to a linear projection pr: $\mathbb{C P}^{2} \backslash\left\{p_{\infty}\right\} \rightarrow \mathbb{C P}^{1},\left(p_{\infty}\right.$ denotes the centre of the projection pr) was introduced in [18] and is a natural generalization of the notion of plane algebraic curves (in [18], Hurwitz curves are called "semi-algebraic" curves). A precise definition of Hurwitz curves can be found, for example, in [7]. In this paper we give another equivalent (see Lemma 1.1) definition of Hurwitz curves as follows. Let $\mathbb{C}_{i}^{2}$ be two copies of the affine plane $\mathbb{C}^{2}, i=1,2$, with coordinates $\left(u_{i}, v_{i}\right), u_{2}=1 / u_{1}$ and $v_{2}=v_{1} / u_{1}$, which cover $\mathbb{C P}^{2} \backslash p_{\infty}$, such that pr is given by $\left(u_{i}, v_{i}\right) \rightarrow u_{i}$ in the charts $\mathbb{C}_{i}^{2}$. A set $\bar{H} \subset \mathbb{C P}^{2} \backslash\left\{p_{\infty}\right\}$, closed in $\mathbb{C P}^{2}$, is called a Hurwitz curve of degree $m$ if, for $i=1,2$,

The first author was partially supported by the DFG-Schwerpunkt "Globale Methoden in der komplexen Geometrie" and the second by the RFBR (02-01-00786) and by the DFG (436 RUS 17/84/03). 
$\bar{H} \cap \mathbb{C}_{i}^{2}$ coincides with the set of zeros of an equation

$$
F_{i}\left(u_{i}, v_{i}\right):=v_{i}^{m}+\sum_{j=0}^{m-1} c_{j, i}\left(u_{i}\right) v_{i}^{j}=0
$$

such that

(i) $F_{i}\left(u_{i}, v_{i}\right)$ is a $C^{\infty}$-smooth complex valued function in $\mathbb{C}^{2}$;

(ii) the function $F_{i}\left(u_{i}, v_{i}\right)$ has only a finite number of critical values, that is, there are finitely many values of $u_{i}$, say $u_{i, 1}, \ldots, u_{i, n_{i}}$, such that the polynomial equation

$$
v_{i}^{m}+\sum_{j=0}^{m-1} c_{j, i}\left(u_{i, 0}\right) v_{i}^{j}=0
$$

has no multiple roots for $u_{i, 0} \notin\left\{u_{i, 1}, \ldots, u_{i, n_{i}}\right\}$;

(iii) if $v_{i, j}$ is a multiple root of equation (2) for $u_{i, j} \in\left\{u_{i, 1}, \ldots, u_{i, n_{i}}\right\}$, then, in a neighbourhood of the point $\left(u_{i, j}, v_{i, j}\right)$ (which we call a critical point of $\bar{H}$ ), the set $\bar{H}$ coincides with the solution a complex analytic equation.

Note that after rescaling $\widetilde{v}_{i}=\varepsilon v_{i}, 0<\varepsilon<<1$, Hurwitz curves become symplectic surfaces in $\mathbb{C P}^{2}$ (see also proof of Theorem 3.1).

More general, one can consider so called topological Hurwitz curves which have cone singularities (see the definition of cone singularities in [7).

A Hurwitz (resp. topological Hurwitz) curve $\bar{H}$ is called irreducible if $\bar{H} \backslash M$ is connected for any finite set $M \subset \bar{H}$, and we say that a Hurwitz curve $\bar{H}$ consists of $k$ irreducible components if

$$
k=\max \#\{\text { connected components of } \bar{H} \backslash M\},
$$

where the maximum is taken over all finite sets $M \subset \bar{H}$.

Let $H$ be an affine Hurwitz curve, that is, $H=\bar{H} \cap\left(\mathbb{C P}^{2} \backslash L_{\infty}\right)$, where $L_{\infty}$ is a line which is a fibre of pr being in general position with respect to $\bar{H}$. Then the fundamental group $\pi_{1}=\pi_{1}\left(\mathbb{C P}^{2} \backslash\left(\bar{H} \cup L_{\infty}\right)\right)$ does not depend on the choice of $L_{\infty}$ and belongs to the class $\mathcal{C}$ of so called $C$-groups.

By definition, a $C$-group is a group together with a finite presentation

$$
G_{W}=<x_{1}, \ldots, x_{m} \mid x_{i}=w_{i, j, k}^{-1} x_{j} w_{i, j, k}, w_{i, j, k} \in W>,
$$

where $W=\left\{w_{i, j, k} \in \mathbb{F}_{m} \mid 1 \leqslant i, j \leqslant m, 1 \leqslant k \leqslant h(i, j)\right\}$ is a subset of elements of the free group $\mathbb{F}_{m}$ (it is possible that $w_{i_{1}, j_{1}, k_{1}}=w_{i_{2}, j_{2}, k_{2}}$ for $\left.\left(i_{1}, j_{1}, k_{1}\right) \neq\left(i_{2}, j_{2}, k_{2}\right)\right)$, generated by free generators $x_{1}, \ldots, x_{m}$ and $h:\{1, \ldots, m\}^{2} \rightarrow \mathbb{Z}$ is some function. Such a presentation is called 
a $C$-presentation $\left(C\right.$, since all relations are conjugations). Let $\varphi_{W}$ : $\mathbb{F}_{m} \rightarrow G_{W}$ be the canonical epimorphism. The elements $\varphi_{W}\left(x_{i}\right) \in G$, $1 \leqslant i \leqslant m$, and the elements conjugated to them are called the $C$ generators of the $C$-group $G$. Let $f: G_{1} \rightarrow G_{2}$ be a homomorphism of $C$-groups. It is called a $C$-homomorphism if the images of the $C$ generators of $G_{1}$ under $f$ are $C$-generators of the $C$-group $G_{2}$. $C$-groups will be considered up to $C$-isomorphisms.

A $C$-presentation (3) is called a Hurwitz $C$-presentation of degree $m$ if for each $i=1, \ldots, m$ the word $w_{i, i, 1}$ coincides with the product $x_{1} \ldots x_{m}$, and a $C$-group $G$ is called a Hurwitz $C$-group of degree $m$ if it possesses a Hurwitz $C$-presentation of degree $m$. In other words, a $C$-group $G$ is a Hurwitz $C$-group of degree $m$ if there are $C$-generators $x_{1}, \ldots, x_{m}$ generating $G$ such that the product $x_{1} \ldots x_{m}$ belongs to the center of $G$. Note that the degree of a Hurwitz $C$-group $G$ is not defined canonically and depends on the Hurwitz $C$-presentation of $G$. Denote by $\mathcal{H}$ the class of all Hurwitz $C$-groups.

Let $\bar{H}$ be a Hurwitz (resp. topological Hurwitz) curve of degree $m$. A Zariski - van Kampen presentation of $\pi_{1}=\pi_{1}\left(\mathbb{C}^{2} \backslash H\right.$ ) (where $\mathbb{C}^{2}=\mathbb{P}^{2} \backslash L_{\infty}$ and $L_{\infty}$, a fibre of pr, is in general position with respect to $\bar{H}$ ) defines on $\pi_{1}$ a structure of a Hurwitz $C$-group of degree $m$ (see [12]), and in [12], it was proved that any Hurwitz $C$-group $G$ of degree $m$ can be realized as the fundamental group $\pi_{1}\left(\mathbb{C}^{2} \backslash H\right)$ for some Hurwitz curve $\bar{H}$ with singularities of the form $w^{m}-z^{m}=0$, deg $\bar{H}=2^{n} m$, where $n$ depends on the Hurwitz $C$-presentation of $G$. Since we consider $C$-groups up to $C$-isomorphisms, the class $\mathcal{H}$ coincides with the class $\left\{\pi_{1}\left(\mathbb{C}^{2} \backslash H\right)\right\}$ of fundamental groups of the complements of affine Hurwitz (resp. topological Hurwitz) curves.

A free group $\mathbb{F}_{n}$ with fixed free generators is a $C$-group and for any $C$-group $G$ the canonical $C$-epimorphism $\nu: G \rightarrow \mathbb{F}_{1}$, sending the $C$ generators of $G$ to the $C$-generator of $\mathbb{F}_{1}$, is well defined. Denote by $N$ its kernel. Note that if all $C$-generators of a $C$-group $G$ are conjugated to each other (such $C$-group is called irreducible), then $N$ coincides with $^{1} G^{\prime}$.

Let $G$ be a $C$-group. The $C$-epimorphism $\nu$ induces the following exact sequence of groups

$$
1 \rightarrow N / N^{\prime} \rightarrow G / N^{\prime} \stackrel{\nu_{*}}{\longrightarrow} \mathbb{F}_{1} \rightarrow 1 \text {. }
$$

\footnotetext{
${ }^{1}$ For a group $G$ we use the standard notation $G^{\prime}$ for its commutator subgroup and $G^{\prime \prime}$ for the commutator subgroup of $G^{\prime}$.
} 
The $C$-generator of $\mathbb{F}_{1}$ acts on $N / N^{\prime}$ by conjugation $\widetilde{x}^{-1} b \widetilde{x}$, where $n \in$ $N$ and $\widetilde{x}$ is one of the $C$-generators of $G$. Denote by $h$ this action and by $h_{\mathbb{C}}$ the induced action on $N / N^{\prime} \otimes \mathbb{C}$. The characteristic polynomial $\Delta(t)=\operatorname{det}\left(h_{\mathbb{C}}-t \mathrm{Id}\right)$ is called the Alexander polynomial of the $C$-group $G$ (if the vector space $N / N^{\prime} \otimes \mathbb{C}$ over $\mathbb{C}$ is infinite dimensional, then, by definition, the Alexander polynomial $\Delta(t) \equiv 0)$. For a (topological) Hurwitz curve $\bar{H}$ the Alexander polynomial $\Delta(t)$ of the group $\pi_{1}=$ $\pi_{1}\left(\mathbb{C}^{2} \backslash H\right)$ is called the Alexander polynomial of $\bar{H}$. Note that the Alexander polynomial $\Delta(t)$ of a (topological) Hurwitz curve $\bar{H}$ does not depend on the choice of the generic line $L_{\infty}$.

Let $G=<x_{1}, \ldots, x_{m} \mid r_{1}, \ldots, r_{n}>$ be a $C$-presentation of a $C$ group $G$ and $\mathbb{F}_{m}$ be the free group freely generated by the $C$-generators $x_{1}, \ldots, x_{m}$. Denote by $\frac{\partial}{\partial x_{i}}$ the Fox derivative $([4)$, that is, the endomorphism of the group ring $\mathbb{Z}\left[\mathbb{F}_{m}\right]$ over $\mathbb{Z}$ of the free group $\mathbb{F}_{m}$ into itself, such that $\frac{\partial}{\partial x_{i}}: \mathbb{Z}\left[\mathbb{F}_{m}\right] \rightarrow \mathbb{Z}\left[\mathbb{F}_{m}\right]$ is a $\mathbb{Z}$-linear map defined by the following properties

$$
\begin{aligned}
& \frac{\partial x_{j}}{\partial x_{i}}=\delta_{i, j} \\
& \frac{\partial u v}{\partial x_{i}}=\frac{\partial u}{\partial x_{i}}+u \frac{\partial v}{\partial x_{i}}
\end{aligned}
$$

for any $u, v \in \mathbb{Z}\left[\mathbb{F}_{m}\right]$. It is well known (it is proved, for example, in [19] in the case of knot groups and generalized to the case of $C$-groups in [10]) that the greatest common divisor of the minors of order $m-1$ in the matrix

$$
\nu_{*}\left(\frac{\partial r_{i}}{\partial x_{j}}\right) \in \operatorname{Mat}_{n \times m}\left(\mathbb{Z}\left[t, t^{-1}\right]\right)
$$

coincides with the Alexander polynomial $\Delta(t)$ of $G$ up to a factor $\pm t^{k}$ invertible in $\mathbb{Z}\left[t, t^{-1}\right]$, where $r_{i}, i=1, \ldots, n$, are the defining relations of $G$ and $\nu_{*}: \mathbb{Z}\left[\mathbb{F}_{m}\right] \rightarrow \mathbb{Z}\left[\mathbb{F}_{1}\right] \simeq \mathbb{Z}\left[t, t^{-1}\right]$ is induced by the canonical $C$-epimorphism $\nu: \mathbb{F}_{m} \rightarrow \mathbb{F}_{1}$.

The properties of the Alexander polynomials of plane algebraic curves and their application to the calculation of the first Betti number of a cyclic covering of the projective plane are well-known (see, for example, [22, [15], 20, 6], 8], 10, 9]). One of the aims of this article is to generalize these results to the case of Hurwitz curves and to apply them to the calculation of the first Betti number of a cyclic covering of the projective plane branched along a Hurwitz curve.

The main results of this article are the following theorems and corollaries.

Theorem 0.1. Let $\bar{H}$ be a (topolgical) Hurwitz curve of degree $d$ and $\Delta(t)$ its Alexander polynomial. Then 
(i) $\Delta(t) \in \mathbb{Z}[t]$;

(ii) $\Delta(0)= \pm 1$;

(iii) the roots of $\Delta(t)$ are $d$-th roots of unity;

(iv) the action of $h_{\mathbb{C}}$ on $\left(N / N^{\prime}\right) \otimes \mathbb{C}$ is semisimple.

Moreover, the Alexander polynomial $\Delta(t)$ of a Hurwitz curve $\bar{H}$ of degree $d$ is a divisor of the polynomial $(t-1)\left(t^{d}-1\right)^{d-2}$ (see Theorem [5.6) and if $\bar{H}$ consists of $k$ irreducible components, then the multiplicity of the root $t=1$ of its Alexander polynomial $\Delta(t)$ is equal to $k-1$ (see Theorem [5.9).

Theorem 0.2. If $\bar{H}$ is an irreducible (topological) Hurwitz curve, then

(i) $\Delta(t)$ is a reciprocal polynomial, i.e. $\Delta(t)=t^{\operatorname{deg} \Delta(t)} \Delta\left(t^{-1}\right)$;

(ii) $\operatorname{deg} \Delta(t)$ is an even number;

(iii) $\Delta(1)=1$.

Corollary 0.3. Let $\bar{H}$ be an irreducible (topological) Hurwitz curve of $\operatorname{deg} \bar{H}=p^{n}$, where $p$ is a prime number. Then

(i) $\Delta(t) \equiv 1$;

(ii) the group $\pi_{1}^{\prime} / \pi_{1}^{\prime \prime}$ is a finite group, where $\pi_{1}=\pi_{1}\left(\mathbb{C}^{2} \backslash H\right)$.

Note also that if $J$ is an almost complex structure in $\mathbb{C P}^{2}$ compatible with the Fubini - Studi symplectic form and if $\bar{H}$ is a $J$-holomorphic curve in $\mathbb{C P}^{2}$ of degree $m$, that is, the class $[\bar{H}]$ equals $m\left[\mathbb{C P}^{1}\right]$ in $H_{2}\left(\mathbb{C P}^{2}, \mathbb{Z}\right)$, then $\pi_{1}=\pi_{1}\left(\mathbb{C P}^{2} \backslash\left(\bar{H} \cup L_{\infty}\right)\right)$ is a Hurwitz $C$-group of degree $m$, where $L_{\infty}$ is one of the $J$-lines being in general position with respect to $\bar{H}$. Indeed, if we chose a pencil of pseudo-holomorphic lines having $L_{\infty}$ as a member, then, by the Zariski-van Kampen Theorem, a presentation of $\pi_{1}$ is defined by a braid monodromy factorization of $\bar{H}$ with respect to the chosen pencil. Therefore $\pi_{1}$ is a $C$-group, and similar to the case of Hurwitz curves, it is easy to show (see the proof of Theorem 6.1 from [12]) that it is a Hurwitz $C$-group of degree $m$. Thus, the Alexander polynomial of a pseudo-holomorphic curve can be defined similarly and has the same properties as in the case of Hurwitz curves.

The homomorphism $\nu: \pi_{1} \rightarrow \mathbb{F}_{1}$, where $\pi_{1}=\pi_{1}\left(\mathbb{C}^{2} \backslash H\right)$ is the fundamental group of the complement of an affine Hurwitz curve, defines an infinite unramified cyclic covering $f=f_{\infty}: X_{\infty}^{\prime} \rightarrow X^{\prime}=\mathbb{C}^{2} \backslash H$. We have $H_{1}\left(X_{\infty}^{\prime}, \mathbb{Z}\right)=N / N^{\prime}$ and the action of $h$ on $H_{1}\left(X_{\infty}^{\prime}, \mathbb{Z}\right)$ coincides with the action of a generator of the covering transformation group of the covering $f_{\infty}$. As it follows from [13], the group $H_{1}\left(X_{\infty}^{\prime}, \mathbb{Z}\right)$ is finitely generated. For any $n \in \mathbb{N}$ denote by $\bmod _{n}: \mathbb{F}_{1} \rightarrow \mu_{n}=\mathbb{F}_{1} /\left\{h^{n}\right\}$ the 
natural epimorphism to the cyclic group $\mu_{n}$ of degree $n$. The covering $f_{\infty}$ can be factorized through the cyclic covering $f_{n}: X_{n}^{\prime} \rightarrow \mathbb{C}^{2} \backslash H$ associated with the epimorphism $\bmod _{n} \circ \nu, f_{\infty}=g_{n} \circ f_{n}$. Since a Hurwitz curve $\bar{H}$ has only analytic singularities, the covering $f_{n}$ can be extended to a smooth map $\bar{f}_{n}: \bar{X}_{n} \rightarrow \mathbb{C P}^{2}$ branched along $\bar{H}$ and, maybe, along $L_{\infty}$ (if $n$ is not a divisor of $\operatorname{deg} \bar{H}$, then $\bar{f}_{n}$ is branched along $\left.L_{\infty}\right)$, where $\bar{X}_{n}$ is a smooth 4 -fold. The action $h$ induces an action $\bar{h}_{n}$ on $\bar{X}_{n}$ and an action $\bar{h}_{n *}$ on $H_{1}\left(\bar{X}_{n}, \mathbb{Z}\right)$.

In section 4, we show (see Theorem 4.1) that any such $\bar{X}_{n}$ can be embedded as a symplectic submanifold to a projective rational 3-fold on which the symplectic structure is given by an integer Kähler form.

Theorem 0.4. Let $\bar{X}_{n}$ be a resolution of singularities of an n-sheeted cyclic covering branched along a Hurwitz curve $\bar{H}$ and, maybe, along $L_{\infty}$ and associated with the epimorphism $\bmod _{n} \circ \nu: \pi_{1} \rightarrow \mathbb{Z} / n \mathbb{Z}$. Then the first Betti number

$$
b_{1}\left(\bar{X}_{n}\right)=\operatorname{dim}_{\mathbb{C}} H_{1}\left(\bar{X}_{n}, \mathbb{C}\right)=r_{n, \neq 1},
$$

where $r_{n, \neq 1}$ is the number of roots of the Alexander polynomial $\Delta(t)$ of the curve $\bar{H}$ which are $n$-th roots of unity not equal to 1.

Theorems 0.1, 0.2, 0.4 and Corollary 0.3 imply the following corollaries.

Corollary 0.5. Let $\bar{X}_{n}$ be a resolution of singularities of an n-sheeted cyclic covering branched along a Hurwitz curve $\bar{H}$ and, maybe, along $L_{\infty}$. If $\operatorname{deg} \bar{H}$ and $n$ are coprime, then $b_{1}\left(\bar{X}_{n}\right)=0$.

Corollary 0.6. Let $\bar{X}_{n}$ be a resolution of singularities of an n-sheeted cyclic covering branched along an irreducible Hurwitz curve $\bar{H}$ and, maybe, along $L_{\infty}$. Then $b_{1}\left(\bar{X}_{n}\right)$ is an even number.

Moreover, we show that for any $k \in \mathbb{N}$, there is an irreducible Hurwitz curve $\bar{H}_{k}$ such that for some $n$ (for example, one can take $n=6$, see Proposition 6.5) a resolution of singularities $\bar{X}_{n}$ of an $n$-sheeted cyclic covering, branched along $\bar{H}_{k}$, has the first Betti number $b_{1}\left(\bar{X}_{k, n}\right)=2 k$. In addition, we show that for any $k \in \mathbb{N}$, there is a Hurwitz curve $\bar{H}_{k}$ consisting of two irreducible components such that the resolution of singularities $\bar{X}_{k, 6}$ of a cyclic covering of the projective plane, branched along $\bar{H}_{k}$, has the first Betti number $b_{1}\left(\bar{X}_{k, 6}\right)=k$. Recall that $b_{1}\left(\bar{X}_{n}\right)$ is always even if $\bar{H}$ is a plane algebraic curve, hence $\bar{H}_{k}$ cannot be algebraic if $k$ is odd. Recall also that Moishezon ([18] proved the existence of an infinite sequence $\bar{H}_{i}$ of irreducible cuspidal Hurwitz curves of degree 54 with exactly 378 cusps and 756 nodes which have pairwise 
distinct braid monodromy type. In particular, they are pairwise nonisotopic, and almost all of them are not isotopic to an algebraic cuspidal curve.

Corollary 0.7. Let $\bar{X}_{n}$ be a resolution of singularities of a cyclic covering of the projective plane branched along a Hurwitz curve $\bar{H}$ consisting of $k$ irreducible components and, maybe, along $L_{\infty}$. If $n$ is divisible by $\operatorname{deg} \bar{H}$, then $b_{1}\left(\bar{X}_{n}\right)=\operatorname{deg} \Delta(t)-k+1$.

Corollary 0.8. Let $\bar{X}_{n}$ be a resolution of singularities of a cyclic covering of the projective plane of any degree $n$ branched along an irreducible Hurwitz curve $\bar{H}$ and, maybe, along $L_{\infty}$. If $\operatorname{deg} \bar{H}=p^{k}$, where $p$ is a prime number, then $b_{1}\left(\bar{X}_{n}\right)=0$.

Corollary 0.9. Let $\bar{X}_{p^{k}}$ be a resolution of singularities of a cyclic covering of the projective plane of degree $p^{k}$ branched along any irreducible Hurwitz curve $\bar{H}$ and, maybe, along $L_{\infty}$, where $p$ is a prime number. Then $b_{1}\left(\bar{X}_{n}\right)=0$.

Note that for any $k \in \mathbb{N}$, we show that there is a Hurwitz curve $\bar{H}_{k}$ consisting of $k+1$ components and which is the branch curve of a 2-sheeted cyclic covering a resolution of singularities $\bar{X}_{k, 2}$ which has $b_{1}\left(\bar{X}_{k, 2}\right)=k$ (see Proposition 6.6) $)$. In particular, in our example the Hurwitz curve $\bar{H}_{1}$ has $\operatorname{deg} \bar{H}_{1}=2^{10}$, the number of singular points of $\bar{H}_{1}$ is equal to $2^{16}$, and all its singular points are of the form $w^{4}-z^{4}=0$.

Recently, Auroux and Katzarkov (see [1], 2]) proved the following theorem. Let $(\bar{X}, \omega)$ be a compact symplectic 4-manifold with symplectic form $\omega$ with class $[\omega] \in H^{2}(\bar{X}, \mathbb{Z})$. Fix an $\omega$-compatible almost complex structure $J$ and the corresponding Riemannian metric $g$. Let $L$ be a line bundle on $\bar{X}$ whose first Chern class is $[\omega]$. Then, for $k>>0$, the line bundle $L^{\otimes k}$ admits many approximately holomorphic sections so that one can choose three of them which give an approximately holomorphic generic covering $f_{k}: \bar{X} \rightarrow \mathbb{C P}^{2}$ of degree $N_{k}=k^{2} \omega^{2}$ branched over a cuspidal Hurwitz curve $\bar{H}_{k}$ (possibly, with negative nodes).

Any such covering $f_{k}: \bar{X} \rightarrow \mathbb{C P}^{2}$ of degree $N_{k}$, branched over a cuspidal Hurwitz curve $\bar{H}$, determines a monodromy $\mu$, that is, an epimorphism $\mu: \pi_{1}\left(\mathbb{C}^{2} \backslash H\right) \rightarrow \Sigma_{N_{k}}$ to the symmetric group $\Sigma_{N_{k}}$ with additinal properties of genericity. On the other hand, any homomorphism $\mu: \pi_{1}\left(\mathbb{C}^{2} \backslash H\right) \rightarrow \Sigma_{N}$, such that $\mu\left(\pi_{1}\right)$ acts transitively on a set consisting of $N$ elements, defines an unramified covering $f: X \rightarrow \mathbb{C}^{2} \backslash H$ of degree $N$. The covering $f$ can be extended to a covering $\tilde{f}: \widetilde{X} \rightarrow \mathbb{C P}^{2}$ branched over the Hurwitz curve $\bar{H}$ and, maybe, over $L_{\infty}$. In this paper 
we prove (see Corollary 3.2 ) that if $\widetilde{X}$ has arbitrary analytic singularities (and if $\bar{H}$ has negative nodes, we assume that the covering space is non-singular over them), then a resolution $\bar{X}$ of singularities of $\widetilde{X}$ can be equipped with a symplectic structure.

The proofs of Theorems $0.1,0.2$ and Corollary 0.3 are given in section 5 and section 6 is devoted to the proof of Theorem 0.4 .

Acknowledgement. The second author would like to express his gratitude to University of Kaiserslautern for its hospitality during the preparation of this paper.

\section{Representation of Hurwitz CuRves as Sections of line BUNDLES}

We begin with the following lemma.

Lemma 1.1. The definitions of Hurwitz curves in $\mathbb{C P}^{2}$ given in [] and in the Introduction are equivalent.

Proof. Recall the definition of Hurwitz curves given in [7]. Let $F_{1}$ be a relatively minimal ruled rational surface, pr $: F_{1} \rightarrow \mathbb{C P}^{1}$ the ruling, $R$ a fiber of pr, and $E_{1}$ the exceptional section, $E_{1}^{2}=-1$. Identify pr : $F_{1} \rightarrow \mathbb{C P}^{1}$ with a linear projection pr $: \mathbb{C P}^{2} \rightarrow \mathbb{C P}^{1}$ with center at a point $p \in \mathbb{C P}^{2}$ ( $p$ is the blow down of $E_{1}$ to the point).

The image $\bar{H}=f(S) \subset F_{1}$ of a smooth map $f: S \rightarrow F_{1} \backslash E_{1}$ of an oriented closed real surface $S$ is called a Hurwitz curve (with respect to pr) of degree $m$ if there is a finite subset $Z \subset \bar{H}$ such that:

(i) $f$ is an embedding of the surface $S \backslash f^{-1}(Z)$ and for any $s \notin Z$, $\bar{H}$ and the fiber $R_{\operatorname{pr}(s)}$ of pr meet at $s$ transversely and with positive intersection number;

(ii) for each $s \in Z$ there is a neighbourhood $U \subset F_{1}$ of $s$ such that $\bar{H} \cap U$ is a complex analytic curve, and the complex orientation of $\bar{H} \cap U \backslash\{s\}$ coincides with the orientation transported from $S$ by $f$

(iii) the restriction of pr to $\bar{H}$ is a finite map of degree $m$.

To show that the definition in the Introduction implies the definition in [7, let us perform several monoidal transforms with centers at singular points of $\bar{H}$ (and at the singularities of the proper transforms of $\bar{H})$ to resolve all singular points of $\bar{H}$. Denote by $\sigma: \widetilde{\mathbb{C P}}^{2} \rightarrow \mathbb{C P}^{2}$ the composition of these monoidal transformations and by $S$ the proper transform of $\bar{H}$. Then $S$ is a smooth real surface and $f=\sigma_{\mid S}$ is a smooth map. To define an orientation on $S$, let us choose an orientation at each non-critical point $p$ of $\operatorname{pr}_{\mid \bar{H}}$ so that the local intersection 
number of $\bar{H}$ and the fibre $R$ passing through $p$ at the point $p$ will be equal to +1 . Obviously, these orientations are compatible for all non-singular points of $\bar{H}$. Since near singular points this orientation coincides with the orientation given by the complex analytic structure (recall that $\bar{H}$ is complex analytic near critical points of $\operatorname{pr}_{\mid \bar{H}}$ ), this orientation can be extended to the preimages of these critical points.

To show that the definition in 7] implies the definition in the Introduction, let us choose a fibre $R$ of pr and put $\mathbb{C}^{2}=F_{1} \backslash\left(R \cup E_{1}\right)$. Let $(u, v)$ be coordinates in $\mathbb{C}^{2}$ such that the restriction of pr is given by $(u, v) \rightarrow u$. Let $\left(u, v_{1}(u)\right), \ldots,\left(u, v_{m}(u)\right)$ be the coordinates of the intersection points of $\bar{H}$ and the fibre $R$ of pr over a non-critical value $u$. Consider

$$
F(u, v)=\prod_{i=1}^{m}\left(v-v_{i}(u)\right) .
$$

Obviously, the function $F(u, v)$, defined everywhere outside the fibres over critical values, is smooth and can be extended to a function on all $\mathbb{C}^{2}$ satisfying the properties of the definition given in the Introduction.

Let a Hurwitz curve $\bar{H}_{0}$ of degree $m$ be given by equations (11). A smooth isotopy $h_{t}: \mathbb{C P}^{2} \times[0,1] \rightarrow \mathbb{C P}^{2} \times[0,1]$ is called a $H$-isotopy if for each $t \in[0,1]$ the image $\bar{H}_{t}=h_{t}\left(\bar{H}_{0}\right)$ is a Hurwitz curve given by equations

$$
v_{i}^{m}+\sum_{j=0}^{m-1} c_{j, i}\left(u_{i}, t\right) v_{i}^{j}=0, \quad i=1,2
$$

$c_{j, i}\left(u_{i}, 0\right)=c_{j, i}\left(u_{i}\right)$ for all $i, j$. (Note that in the definition of $H$-isotopy given in [7], it is assumed that the number of critical values of $\bar{H}_{t}$ does not depend on $t$.) It is easy to see that if $\bar{H}_{0}$ and $\bar{H}_{1}$ are $H$ isotopic and a line $L_{\infty}$ is generic with respect to both Hurwitz curves $\bar{H}_{0}$ and $\bar{H}_{1}$, then $\mathbb{C}^{2} \backslash H_{0}$ and $\mathbb{C}_{2} \backslash H_{1}$ are diffeomorphic.

Denote by $p_{\infty}=\mathbb{C P}^{2} \backslash\left(\mathbb{C}_{1}^{2} \cup \mathbb{C}_{2}^{2}\right)$ the center of the projection pr. In what follows we will assume that the fibre of pr over $u_{2}=0$ is generic with respect to $\bar{H}_{0}$. Denote it by $L_{\infty}$. Obviously, there is a smooth $H$-isotopy $h_{t}$ identical outside a small neighbourhood $U$ of $L_{\infty}$ such that the function $F_{2}\left(u_{2}, v_{2}, 1\right)$ defining $\bar{H}_{1}=h_{1}\left(\bar{H}_{0}\right)$ in $\mathbb{C}_{2}^{2}$ coincides with the function $v_{2}^{m}-1$ at the points $\left(u_{2}, v_{2}\right)$ with $\left|u_{2}\right|<\varepsilon$ for some $\varepsilon>0$. In what follows we will assume that

$$
\begin{aligned}
& L_{\infty} \text { is given by } u_{2}=0 \text { and } \bar{H} \text { is given by the equation } \\
& v_{2}^{m}-1=0 \text { in a neighbourhood of } L_{\infty} .
\end{aligned}
$$


Let $u_{1, j}$ be a critical value of a Hurwitz curve $\bar{H}_{0}$ of degree $m$ given in $\mathbb{C}_{1}^{2}$ by equation $F_{1}\left(u_{1}, v_{1}\right)=0$, that is, the number of the different roots of the equation

$$
F_{1}\left(u_{1, j}, v_{1}, 0\right)=0
$$

is less than $m$, and let $v_{1, j_{0}}$ be a root of equation (17) of multiplicity one. It is obvious that there is a smooth $H$-isotopy $h_{t}$ identical outside a small neighbourhood $U=\left\{\left|u_{1}-u_{1, j}\right|<\varepsilon\right\}$ such that the function $F_{1}\left(u_{1}, v_{1}, 1\right)$ defining $\bar{H}_{1}=h_{1}\left(\bar{H}_{0}\right)$ in $\mathbb{C}_{1}^{2}$ is such that $v_{1}=v_{1, j_{0}}$ is a root of the equation $F_{1}\left(u_{1}, v_{1}, 1\right)=0$ for all $u_{1}$ such that $\left|u_{1}-u_{1, j}\right|<\varepsilon_{1}$ for some positive $\varepsilon_{1}<\varepsilon$. Therefore, in what follows we can (and we will) assume that if $u_{1, j}$ is a critical value of $\bar{H}$, then

$$
\begin{aligned}
& \text { there is an } \varepsilon>0 \text { such that } F_{1}\left(u_{1}, v_{1}\right) \text { defining } \bar{H} \text { is } \\
& \text { analytic at }\left(u_{1}, v_{1}\right) \text { for }\left|u_{1}-u_{1, j}\right|<\varepsilon \text { and all } v_{1} \text {. }
\end{aligned}
$$

Let us consider a line bundle $p: \mathcal{L}(k) \rightarrow \mathbb{C P}^{2}$ associated with the sheaf $\mathcal{O}_{\mathbb{C P}^{2}}(k)$. Recall its definition. The projective plane $\mathbb{C P}^{2}$ with homogeneous coordinates $\left(z_{0}: z_{1}: z_{2}\right)$ is covered by three charts $\mathbb{C}_{i}^{2}$, $i=1,2,3$, isomorphic to $\mathbb{C}^{2}$, with coordinates $\left(u_{i}, v_{i}\right), u_{1}=z_{1} / z_{0}$, $v_{1}=z_{2} / z_{0}, u_{2}=z_{0} / z_{1}, v_{2}=z_{2} / z_{1}, u_{3}=z_{0} / z_{2}, v_{3}=z_{1} / z_{2}$. The bundle $\mathcal{L}(k)$ is covered by three charts $W_{i}=\mathbb{C}_{i}^{2} \times \mathbb{C}_{i}^{1}$ with the third coordinate $w_{i}, w_{1}=w_{2} / u_{2}^{k}, w_{1}=w_{3} / u_{3}^{k}, w_{2}=w_{3} / v_{3}^{k}$, and the restriction of $p_{\mid W_{i}}$ coincides with the projection to the first factor.

Lemma 1.2. The functions $w_{i}=F_{i}\left(u_{i}, v_{i}\right), i=1,2$, defining $\bar{H}$ define a smooth section s of $\mathcal{L}(m)$ over $\mathbb{C P}^{2} \backslash\left\{p_{\infty}\right\}$.

Proof. In $\mathbb{C}_{1}^{2} \cap \mathbb{C}_{2}^{2}$ we have

$$
\begin{aligned}
F_{1}\left(u_{1}, v_{1}\right)= & v_{1}^{m}+\sum_{j=0}^{m-1} c_{j, 1}\left(u_{1}\right) v_{1}^{j}=\left(\frac{v_{2}}{u_{2}}\right)^{m}+\sum_{j=0}^{m-1} c_{j, 1}\left(\frac{1}{u_{2}}\right)\left(\frac{v_{2}}{u_{2}}\right)^{j}= \\
& \left(\frac{1}{u_{2}}\right)^{m}\left(v_{2}^{m}+\sum_{j=0}^{m-1} c_{j, 1}\left(\frac{1}{u_{2}}\right) u_{2}^{m-j} v_{2}^{j}\right) .
\end{aligned}
$$

The functions

$$
F_{2}\left(u_{2}, v_{2}\right)=v_{2}^{m}+\sum_{j=0}^{m-1} c_{j, 2}\left(u_{2}\right) v_{2}^{j}
$$

and

$$
v_{2}^{m}+\sum_{j=0}^{m-1} c_{j, 1}\left(\frac{1}{u_{2}}\right) u_{2}^{m-j} v_{2}^{j}
$$


coincide with each other, since they are smooth and for almost all (except a finite number of) values $u_{2,0}$ of $u_{2}$ the polynomials

$$
v_{2}^{m}+\sum_{j=0}^{m-1} c_{j, 2}\left(u_{2,0}\right) v_{2}^{j}
$$

and

$$
v_{2}^{m}+\sum_{j=0}^{m-1} c_{j, 1}\left(\frac{1}{u_{2,0}}\right) u_{2,0}^{m-j} v_{2}^{j}
$$

have the same sets of roots.

Lemma 1.3. Let $f_{0}: S^{3} \rightarrow \mathbb{C}^{*}$ be a smooth function on $S^{3}=\{(u, v) \in$ $\left.\mathbb{C}^{2} \mid u \bar{u}+v \bar{v}=\varepsilon^{2}\right\}, 0<\varepsilon<<1$, such that $f_{0}$ coincides with the function $v^{m}-1$ in a neighbourhood $U \subset S^{3}$ of the circle $u=0$. Then there is a smooth function $F: S^{3} \times[0,1] \rightarrow \mathbb{C}^{*}$ such that

(i) $F(u, v, 0)=f_{0}(u, v)$;

(ii) $F(u, v, t)=v^{m}-1$ for $(u, v) \in U$ and $t \in[0,1]$;

(iii) $F(u, v, 1)=v^{m}-1$.

Proof. Since $S^{3}$ is simply connected, there is a lift $\widetilde{f}_{0}: S^{3} \rightarrow \widetilde{\mathbb{C}}^{*}$ of the function $f_{0}$ such that $f_{0}=e \circ \widetilde{f}_{0}$, where $\widetilde{\mathbb{C}}^{*}$ is the complex plane $\mathbb{C}$ with complex coordinate $x$ and $e: \widetilde{\mathbb{C}}^{*} \rightarrow \mathbb{C}^{*}$ is the universal covering given by $y=e^{x}$. Without loss of generality one can assume that $\widetilde{f}_{0}(0, \varepsilon)=\ln \left(1-\varepsilon^{m}\right)+\pi i$. Denote by $f_{1}: S^{3} \rightarrow \mathbb{C}^{*}$ the function $v^{m}-1$ and by $\widetilde{f}_{1}: S^{3} \rightarrow \widetilde{\mathbb{C}}^{*}$ its lift such that $\widetilde{f}_{1}(0, \varepsilon)=\ln \left(1-\varepsilon^{m}\right)+\pi i$. Therefore we have $\widetilde{f}_{0 \mid U} \equiv \widetilde{f}_{1 \mid U}$.

Consider a function $\widetilde{F}: S^{3} \times[0,1] \rightarrow \mathbb{C}$ given by

$$
x=t \widetilde{f}_{1}(u, v)+(1-t) \widetilde{f}_{0}(u, v) .
$$

Obviously, the function $F=e \circ \widetilde{F}$ has the desired properties.

Lemma 1.4. There is a real number $\varepsilon_{1}, 0<\varepsilon_{1}<<1$, and a smooth section $\bar{s}_{m}$ of $\mathcal{L}(m)$ over $\mathbb{C P}^{2}$ such that

(i) $\bar{H} \subset \mathbb{C P}^{2} \backslash B\left(\varepsilon_{1}\right)$, where $B\left(\varepsilon_{1}\right)=\left\{u_{3} \bar{u}_{3}+v_{3} \bar{v}_{3} \leqslant \varepsilon_{1}^{2}\right\}$ is a ball in $\mathbb{C P}^{2}$ with center at $p_{\infty}$;

(ii) over $\mathbb{C P}^{2} \backslash B\left(\varepsilon_{1}\right)$ the section $\bar{s}_{m}$ coincides with the section $s$ from Lemma 1.2;

(iii) $\bar{s}_{m}$ is complex analytic in a neighbourhood of the line $L_{\infty}$. 
Proof. By the definition of Hurwitz curves, there is a ball $B\left(\varepsilon_{1}\right)=$ $\left\{u_{3} \bar{u}_{3}+v_{3} \bar{v}_{3} \leqslant \varepsilon_{1}^{2}\right\}$ for some positive $\varepsilon_{1}$ such that $\bar{H} \subset \mathbb{C P}^{2} \backslash B\left(\varepsilon_{1}\right)$.

The line bundle $\mathcal{L}_{m}$ is trivial over $B\left(\varepsilon_{1}\right)$. Therefore the restriction of the section $s$ from Lemma 1.2 to $\partial B\left(\varepsilon_{1}\right)=S^{3}$ defines a function $f_{0}$ : $S^{3} \rightarrow \mathbb{C}^{*}$. Denote by $F: S^{3} \times[0,1] \rightarrow \mathbb{C}^{*}$ a function existence of which is proved in Lemma 1.3 (in the notations of Lemma 1.3. we put $u=u_{3}$, $v=v_{3}$ and $\left.\varepsilon=\varepsilon_{1}\right)$. Let us choose $\varepsilon_{2}<\varepsilon_{1}$ and a smooth monotone function $r:\left[\varepsilon_{2}, \varepsilon_{1}\right] \rightarrow[0,1]$ such that $r\left(\varepsilon_{1}\right)=0$ and $r\left(\varepsilon_{2}\right)=1$. Put $h: B\left(\varepsilon_{1}, \varepsilon_{2}\right)=B\left(\varepsilon_{1}\right) \backslash B\left(\varepsilon_{2}\right) \rightarrow \partial B\left(\varepsilon_{1}\right)$ to be the map given by

$$
h\left(u_{3}, v_{3}\right)=\left(\frac{\varepsilon_{1} u_{3}}{\sqrt{u_{3} \bar{u}_{3}+v_{3} \bar{v}_{3}}}, \frac{\varepsilon_{1} v_{3}}{\sqrt{u_{3} \bar{u}_{3}+v_{3} \bar{v}_{3}}}\right)
$$

$\widetilde{F}\left(u_{3}, v_{3}, t\right)=h^{*}(F)$, and

$$
\bar{F}\left(u_{3}, v_{3}\right)=\left(\frac{\sqrt{u_{3} \bar{u}_{3}+v_{3} \bar{v}_{3}}}{\varepsilon_{1}}\right)^{m}\left(\widetilde{F}\left(u_{3}, v_{3}, r\left(\sqrt{u_{3} \bar{u}_{3}+v_{3} \bar{v}_{3}}\right)\right)+1\right)-1 .
$$

Then the section $\widetilde{s}$, defined as follows,

$$
\widetilde{s}(p)= \begin{cases}s(p) & \text { for } p \in \mathbb{C P}^{2} \backslash B\left(\varepsilon_{1}\right) \\ \bar{F}\left(u_{3}, v_{3}\right) & \text { for } p \in B\left(\varepsilon_{1}\right) \backslash B\left(\varepsilon_{2}\right) \\ v_{3}^{m}-1 & \text { for } p \in B\left(\varepsilon_{2}\right),\end{cases}
$$

satisfies all conditions of Lemma 1.4, except that, possibly, it is not smooth, but only continuous at the points from $B=\left(\partial B\left(\varepsilon_{1}\right) \cup \partial B\left(\varepsilon_{2}\right)\right) \backslash$ $U$, where $U$ is a neighbourhood of $L_{\infty}$. By standard theorems from analysis, there is a smooth section $\bar{s}_{m}$ close enough to $\widetilde{s}$ which coincides with $\widetilde{s}$ outside a small enough neighbourhood $V$ of $B$ such that $\bar{V} \cap$ $\left(\bar{H} \cup L_{\infty}\right)=\emptyset$, where $\bar{V}$ is the closure of $V$.

\section{Symplectic VARIEties With ANALYTiC Singularities}

Let $Y$ be a projective complex manifold, $\operatorname{dim}_{\mathbb{C}} Y=n$ and $\omega$ a Kähler form on $Y,[\omega] \in H^{2}(Y, \mathbb{Z})$. Consider $(Y, \omega)$ as a symplectic manifold, $\operatorname{dim}_{\mathbb{R}} Y=2 n$. A closed subvariety $X$ of $Y$ is called a symplectic variety with analytic singularities if there are open subsets $U_{0} \subset U \subset Y$ such that the closure $\bar{U}_{0}$ in $Y$ is a subset of $U, X \cap U$ is a complex analytic subset in $U$ and $X \backslash \bar{U}_{0}$ is a smooth symplectic submanifold. Denote by Sing $X$ the set of points of $X$ in which $X$ is not smooth. Then $\operatorname{Sing} X$ is a projective algebraic subvariety of $Y$.

Lemma 2.1. Let $X$ be a symplectic variety with analytic singularities in a projective complex manifold $Y$ with Kähler form $\omega$. Let $Z \subset$ Sing $X$ be a nonsingular projective subvariety of $Y, \sigma: \bar{Y} \rightarrow Y$ the monoidal transformation of $Y$ with center in $Z$, and $\bar{X}$ be the proper 
transform of $X$. Then there is a Kähler form $\bar{\omega}$ on $\bar{Y}$ such that $\bar{X}$ is a symplectic subvariety with analytic singularities of $(\bar{Y}, \bar{\omega})$.

Proof. The manifold $\bar{Y}$ is projective algebraic. Consider an embedding $i: \bar{Y} \hookrightarrow \mathbb{C P}^{N}$ to some projective space and denote by $\varphi=i \circ \sigma^{-1}$ a rational map from $Y$ to $\mathbb{C} \mathbb{P}^{N}$. Let $\Gamma \subset Y \times \mathbb{C P}^{N}$ be the closure of the graph of $\varphi$ and $p_{i}, i=1,2$, be the projections from $Y \times \mathbb{C P}^{N}$ to the factors. The morphisms $i$ and $\sigma$ define the morphism $\sigma \times i: \bar{Y} \rightarrow \Gamma \subset$ $Y \times \mathbb{C P}^{N}$. Since the composition $p_{2} \circ(\sigma \times i): \bar{Y} \rightarrow \bar{Y}$ is an isomorphism, $p_{2 \mid \Gamma}: \Gamma \rightarrow \bar{Y}$ is an isomorphism too. Moreover, if we identify $\bar{Y}$ with $\Gamma$ by means of $p_{2 \mid \Gamma}$, then $p_{1 \mid \Gamma}$ coincides with $\sigma$.

Denote by $\Omega=\Omega_{N}$,

$$
\Omega_{N}=\frac{i}{\left(\sum_{j=0}^{N} \bar{z}_{j} z_{j}\right)^{2}} \sum_{k=0}^{N} \sum_{j \neq k}\left(\bar{z}_{j} z_{j} d z_{k} \wedge d \bar{z}_{k}-\bar{z}_{j} z_{k} d z_{j} \wedge d \bar{z}_{k}\right),
$$

the Fubini - Studi symplectic form on $\mathbb{C P}^{N}$. It is a Kähler integer form. Consider the restriction of a form $\omega_{\varepsilon}=p_{1}^{*}(\omega)+\varepsilon p_{2}^{*}(\Omega)$ to $\Gamma$. It is a Kähler form on $\Gamma$ for each $\varepsilon>0$.

Choose open neighbourhoods $V_{0} \subset V \subset Y$ of $\operatorname{Sing} X$ such that $V \cap X$ is an analytic subvariety and the closure $\bar{V}_{0}$ of $V_{0}$ in $Y$ is a subset of $V$. Denote by $X_{0}=X \backslash V_{0}$. It is compact and $\sigma_{\mid X_{0}}^{-1}: X_{0} \rightarrow \bar{X}_{0}=\sigma^{-1}\left(X_{0}\right)$ is an isomorphism. Therefore $\bar{X}_{0}$ is compact.

Evidently, the restriction of $\omega_{\varepsilon}$ to $\Gamma \cap p_{1}^{-1}(V)$ is a symplectic form at each nonsingular point of $\Gamma \cap p_{1}^{-1}(V)$ for all $\varepsilon>0$, since $\Gamma \cap p_{1}^{-1}(V)$ is an analytic set in $p_{1}^{-1}(V)$. Since the restriction of $\omega$ to $X_{0}$ is a symplectic form at each point of $X_{0}$ and $\bar{X}_{0}$ is compact, we can choose $\varepsilon$ to be small enough, so that the restriction of $\omega_{\varepsilon}=p_{1}^{*}(\omega)+\varepsilon p_{2}^{*}(\Omega)$ to $\bar{X}_{0}=\Gamma \cap p_{1}^{-1}\left(X_{0}\right)$ is a symplectic form at each point of $\bar{X}_{0}$. If we take $\varepsilon=\frac{m}{n}$ rational, then $n \omega_{\varepsilon}$ is an integer form.

\section{Symplectness of COVERings of the PROJective Plane BRANCHED ALONG HURWITZ CURVES}

In this section we use the notations and assumptions of section 1 .

Let $\bar{H}$ be a Hurwitz curve, possibly with negative nodes, that is, in a neighbourhood $U$ of each critical point $p$, it is either given by an analytic equation or $\bar{H} \cap U$ consists of two smooth branches meeting transversely at $p$ with intersection number -1 , and each branch of $\bar{H} \cap U$ meets the fiber $\operatorname{pr}^{-1}(\operatorname{pr}(p))$ transversely at $p$ with intersection number equals +1 .

We fix a point $p \in \mathbb{C P}^{2} \backslash\left(\bar{H} \cup L_{\infty}\right)$. Consider the fundamental group $\pi_{1}=\pi_{1}\left(\mathbb{C}^{2} \backslash H, p\right)$ of the complement of the affine Hurwitz curve 
$H=\left(\mathbb{C P}^{2} \backslash L_{\infty}\right) \cap \bar{H}$. Let us choose a point $x \in \bar{H} \backslash$ Sing $\bar{H}$ and consider a line $L \subset \mathbb{C}^{2}$ meeting $H$ transversely at $x$. Let $\gamma \subset L$ be a circle of small radius with center at $x$. The choice of an orientation on $\mathbb{C}^{2}$ defines an orientation on $\gamma$. Let $\Gamma$ be a loop consisting of a path $l$ in $\mathbb{C}^{2} \backslash H$, joining $p$ with a point $q \in \gamma$, the loop $\gamma$ (with positive direction) starting and ending at $q$, and a return path to $p$ along $l$ in the opposite direction. Such a loop $\Gamma$ (and the corresponding element in $\pi_{1}$ ) is called a geometric generator (with center at $x$ ) of the fundamental group $\pi_{1}=\pi_{1}\left(\mathbb{C}^{2} \backslash H, p\right)$. It is well known that $\pi_{1}$ is generated by geometric generators.

For each critical point $s_{i}$ of $H$ let us choose a neighbourhood $U_{i} \subset \mathbb{C}^{2}$ such that $H \cap U_{i}$ is given (in local coordinates in $U_{i}$ ) by an analytic equation or, if $s_{i}$ is a negative node, then it consists of two smooth branches meeting transversely at $p$. Note that if $s_{i}$ is a negative node, then $\pi_{1}\left(U_{i} \backslash H, p_{i}\right)$ is isomorphic to $\mathbb{Z} \oplus \mathbb{Z}$ and generated by two commuting geometric generators.

Choose smooth paths $\gamma_{i}$ lying in $\mathbb{C}^{2} \backslash H$ and connecting the points $p_{i}$ with $p$. This choice defines homomorphisms $\psi_{i}: \pi_{1}\left(U_{i} \backslash H, p_{i}\right) \rightarrow \pi_{1}$. We call $\psi_{i}\left(\pi_{1}\left(U_{i} \backslash H, p_{i}\right)\right)=G_{i}$ the local fundamental group of the singular point $s_{i}$. The local fundamental groups are defined uniquely up to conjugation in $\pi_{1}$.

Consider a homomorphism $\mu: \pi_{1} \rightarrow \Sigma_{N}$ from the fundamental group $\pi_{1}=\pi_{1}\left(\mathbb{C}^{2} \backslash H\right)$ of the complement of the affine Hurwitz curve $H=$ $\left(\mathbb{C P}^{2} \backslash L_{\infty}\right) \cap \bar{H}$ to the symmetric group $\mathfrak{S}_{N}$ such that its image $\operatorname{Im} \mu$ acts transitively on a set consisting of $N$ elements.

Let $s_{i}$ be a negative node of $H$. As it was mentioned above, the local fundamental group $G_{i}$ is generated by two commuting geometric generators, say $\Gamma_{i, 1}$ and $\Gamma_{i, 2}$. Denote by $N_{i, j}=\left\{1 \leqslant n \leqslant N \mid \mu\left(\Gamma_{i, j}\right)(n) \neq n\right\}$. We say that $\mu$ is good at the negative node $s_{i}$ if $N_{i, 1} \cap N_{i, 2}=\emptyset$. The homomorphism $\mu$ is called a monodromy of degree $N$ if it is good at all negative nodes.

The homomorphism $\mu$ defines an unramified covering $f=f_{\mu}: Y \rightarrow$ $\mathbb{C}^{2} \backslash H$ of degree $N$. This covering can be extended to a finite ramified covering $\widetilde{f}: \widetilde{Y} \rightarrow \mathbb{C P}^{2}$ branched along $\bar{H}$ and, maybe, along $L_{\infty}$.

To describe this extension, consider a geometric generator $\Gamma$ with center at $x \in H \backslash$ Crit $H$, where Crit $H$ is the set of critical points of $H$. The image $\mu(\Gamma)$ in $\Sigma_{N}$ is a product of cyclic permutations $\sigma_{1}, \ldots, \sigma_{n_{x}}$ (it is possible that the orders of some $\sigma_{l}$ are equal to one). Let $\sigma_{l}=$ $\left(n_{1, l}, \ldots, n_{r_{l}, l}\right)$ be a permutation of order $r_{l}$, where $1 \leqslant n_{j, l} \leqslant N$, then the number of the preimages $\tilde{f}^{-1}(x)$ is equal to $n_{x}$ and each point $y$ from $\tilde{f}^{-1}(x)$ corresponds to a cyclic permutation $\sigma_{l}$. Near the point 
$y_{l}$ corresponding to a cyclic permutation $\sigma_{l}$, the covering $\tilde{f}$ is a cyclic covering of degree $r_{l}$ branched along $H$ and it is locally isomorphic to a subvariety of $\mathbb{C}^{3}$ given by $w^{r_{l}}=v-v_{j}(u)$, where $v-v_{j}(u)=0$ is a local equation of $\bar{H}$ at the point $x$ (see (15)). These local isomorphisms define on $\widetilde{Y}$ a structure of a smooth manifold at each point $y$ lying over $H \backslash$ Crit $H$.

Let $s_{i} \in$ Crit $H$ be a negative node. As it was mentioned above, the local fundamental group $G_{i}$ is generated by two geometric generators $\Gamma_{i, 1}$ and $\Gamma_{i, 2}$. The images $\mu\left(\Gamma_{i, j}\right)$ in $\Sigma_{N}$ are products of cyclic permutations $\sigma_{1, i, j}, \ldots, \sigma_{k_{i, j}, i, j}$. Let $\sigma_{e, i, j}=\left(n_{1, l, i, j}, \ldots, n_{r_{l, i, j}, l, i, j}\right)$ be a permutation of order $r_{l, i, j}$. Put $N_{i, 1,2}=\left\{1 \leqslant n \leqslant N \mid \mu\left(\Gamma_{i, 1}\right)(n)=\right.$ $n$ and $\left.\mu\left(\Gamma_{i, 2}\right)(n)=n\right\}$. Since $\mu$ is a monodromy, the set $\widetilde{f}^{-1}\left(s_{i}\right)$ is in one-to-one correspondence with the union of the cyclic permutations $\sigma_{l, i, j}, j=1,2$, of orders bigger than one and the set $N_{i, 1,2}$. Moreover, if $y \in \widetilde{f}^{-1}\left(s_{i}\right)$ corresponds to an element from $N_{i, 1,2}$, then $\widetilde{f}$ is an isomorphism of a neighbourhood $V$ of $y$ and its image $\widetilde{f}(V)$, and if $y \in \widetilde{f}^{-1}\left(s_{i}\right)$ corresponds to a cyclic permutation $\sigma_{l, i, j}$ of order bigger than one, then the restriction of $\tilde{f}$ to a neighbourhood of $y$ is a cyclic covering of a neighbourhood of $s_{i}$ of degree $r_{l, i, j}$ branched along the $j$-th branch of the negative node. It is locally isomorphic to a subvariety of $\mathbb{C}^{3}$ given by $w^{r_{l, i, j}}=v-v_{j}(u)$, where $v-v_{j}(u)=0$ is a local equation of the $j$-th branch of $\bar{H}$ at the point $s_{i}$. These local isomorphisms define on $\widetilde{Y}$ a structure of smooth manifold at each point $y \in \widetilde{f}^{-1}\left(s_{i}\right)$.

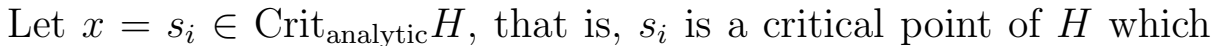
is not a negative node. Then over a small neighbourhood $U$ of $s_{i}$ in which $H$ is given by an analytic equation, the preimage $\bar{f}^{-1}(U)$ is the disjoint union of $n_{s_{i}}$ open neighbourhoods being in one-to-one correspondence with the orbits of the action of $\mu\left(G_{i}\right)$ on the set consisting of $N$ elements. By the theorem of Grauert, Remmert and Stein (for a proof see 21]) we know that, over a neighbourhood $U$ of $s_{i}$ the variety $\widetilde{Y}$ can be equipped with the structure of a two dimensional complex analytic variety.

By assumption, in a neighbourhood $U$ of $L_{\infty}$ (where $U=\mathbb{C P}^{2} \backslash B(R)$ and $B(R) \subset \mathbb{C}_{1}^{2}$ is a ball of big radius $R$ ) the curve $\bar{H}$ coincides with the algebraic curve $\bar{C} \subset \mathbb{C P}^{2}$ of degree $m$ given in $U$ by the equation $v_{2}^{m}-1=0$. If we choose the base point $p$ to lie in $U$, we can consider a Zariski - van Kampen presentations of $\pi_{1}$ and of the fundamental group $\widetilde{\pi}_{1}=\pi_{1}\left(\mathbb{C P}^{2} \backslash\left(\bar{C} \cup L_{\infty}\right), p\right)$ having the same sets of generators and it is easy to see that these presentations define an epimorphism $e: \widetilde{\pi}_{1} \rightarrow \pi_{1}$. The composition $\mu \circ e$ defines a ramified covering $\widetilde{g}: \widetilde{Z} \rightarrow$ 
$\mathbb{C P}^{2}$ branched along $\bar{C}$ and, maybe, along $L_{\infty}$. It is easy to see that the coverings $\widetilde{f}$ and $\widetilde{g}$ are isomorphic over $U$. Therefore the variety $\widetilde{f}^{-1}(U)$ can be identified with $\widetilde{g}^{-1}(U)$ by means of an isomorphism $h: \widetilde{f}^{-1}(U) \rightarrow \widetilde{g}^{-1}(U)$ so that $\tilde{f}^{-1}(U)$ can also be considered as a complex analytic variety. Let $i: \widetilde{Z} \hookrightarrow \mathbb{C P}^{m_{\infty}}$ be an embedding such that $\widetilde{g}$ is defined by the projection $\left(z_{0}^{\prime}: z_{1}^{\prime}: \cdots: z_{m_{\infty}}^{\prime}\right) \rightarrow\left(z_{0}^{\prime}: z_{1}^{\prime}: z_{2}^{\prime}\right)$. Put $z_{j}=h^{*}\left(z_{j}^{\prime}\right), j=3, \ldots, m_{\infty}$ and

$$
w_{j, \infty}^{\prime}=\frac{z_{j}}{z_{0}}, \quad j=3, \ldots, m_{\infty} .
$$

Theorem 3.1. Let $\bar{H}$ be a Hurwitz curve with negative nodes, $\mu$ : $\pi_{1}\left(\mathbb{C}^{2} \backslash H\right) \rightarrow \Sigma_{N}$ a monodromy, and $\tilde{f}: Y \rightarrow \mathbb{C P}^{2}$ the covering associated with the monodromy $\mu$. Then $\widetilde{Y}$ can be embedded into some projective space $\mathbb{C P}^{M}$ as a symplectic subvariety with analytic singularities.

Proof. Below, for each point $p \in \mathbb{C}_{1}^{2}$, we denote by $V_{p} \subset U_{p}$ small balls $V_{p}=\left\{\left|u_{1}-u_{1}(p)\right|^{2}+\left|v_{1}-v_{1}(p)\right|^{2}<\delta_{1}\right\}$ and $U_{p}=\left\{\left|u_{1}-u_{1}(p)\right|^{2}\right.$ $\left.+\left|v_{1}-v_{1}(p)\right|^{2}<\delta_{2}\right\}$ of radii $\delta_{1}$ and $\delta_{2}, 0<\delta_{1}<\delta_{2}<<1$, and by $\rho_{p}: \mathbb{C P}^{2} \rightarrow \mathbb{R}$ we denote a smooth non negative function such that $\rho_{p \mid V_{p}} \equiv 1$ and $\rho_{p \mid \mathbb{C P}^{2} \backslash U_{p}} \equiv 0$.

To construct the desired embedding, let us choose two open coverings $\left\{U_{i}\right\}$ and $\left\{V_{i}\right\}$ of $\mathbb{C P}^{2}$ as follows.

For each point $s_{i} \in \mathrm{Crit}_{\text {analytic }} \bar{H}$ which is not a negative node, let us choose small neighbourhoods $V_{s_{i}}^{\prime} \subset V_{s_{i}} \subset U_{s_{i}} \subset U_{s_{i}}^{\prime}$ such that

$\left(c_{1}\right)$ the curve $U_{s_{i}}^{\prime} \cap \bar{H}$ is analytic in $U_{s_{i}}^{\prime}$;

$\left(c_{2}\right)$ the preimage $\tilde{f}^{-1}\left(U_{s_{i}}^{\prime}\right)$ splits into the disjoint union of neighbourhoods of the points $y_{i, j} \in \widetilde{f}^{-1}\left(s_{i}\right)$;

$\left(c_{3}\right)$ the radius of the ball $V_{s_{i}}^{\prime}\left(\operatorname{resp} . U_{s_{i}}\right)$ is strictly less than the radius of $V_{s_{i}}\left(\operatorname{resp} . U_{s_{i}}^{\prime}\right)$.

Let $V_{\infty}^{\prime} \subset V_{\infty} \subset U_{\infty} \subset U_{\infty}^{\prime}$ be open neighbourhoods of $L_{\infty}$ such that

$\left(c_{4}\right)$ in $U_{\infty}^{\prime}$, the Hurwitz curve $\bar{H}$ coincides with the curve $\bar{C}$ given in $U_{\infty}^{\prime}$ by equation $v_{2}^{m}-1=0$;

$\left(c_{5}\right) U_{\infty}^{\prime} \cap V_{s_{i}}^{\prime}=\emptyset$ for all neighbourhoods $V_{s_{i}}^{\prime}$ of singular points $s_{i}$ chosen above;

$\left(c_{6}\right)$ the closure $\bar{V}_{\infty}^{\prime} \subset V_{\infty}$ and the closure $\bar{U}_{\infty} \subset U_{\infty}^{\prime}$.

Let $\rho_{\infty}: \mathbb{C P}^{2} \rightarrow \mathbb{R}$ be a smooth non negative function such that $\rho_{\infty \mid V_{\infty}} \equiv 1$ and $\rho_{\infty \mid \mathbb{C P}^{2} \backslash U_{\infty}} \equiv 0$.

Let us add the neighbourhoods $U_{\infty}$ and $V_{\infty}$ to the sets $\left\{U_{s_{i}}\right\}$ and $\left\{V_{s_{i}}\right\}$ chosen above. 
Next, for each point $p \in \mathbb{C}_{1}^{2} \backslash\left(\left(\cup V_{s_{i}}\right) \cup V_{\infty}\right)$, we can find open neighbourhoods $V_{p} \subset U_{p}$ of $p$ such that

$\left(c_{7}\right) U_{p} \cap V_{s_{i}}^{\prime}=\emptyset$ and $U_{p} \cap V_{\infty}^{\prime}=\emptyset$ for the neighbourhoods $V_{s_{i}}^{\prime}$ and $V_{\infty}^{\prime}$ chosen above;

$\left(c_{8}\right)$ the preimage $\tilde{f}^{-1}\left(U_{p}\right)$ splits into the disjoint union of neighbourhoods of the points $y_{j} \in \widetilde{f}^{-1}(p)$.

$\left(c_{9}\right)$ if $p \in U_{s_{i}}^{\prime}$ (resp. $p \in U_{\infty}^{\prime}$ ) for some neighbourhood $U_{s_{i}}^{\prime}$ (resp. $\left.U_{\infty}^{\prime}\right)$ chosen above, then $U_{p} \subset U_{s_{i}}^{\prime}$ (resp. $\left.U_{p} \subset U_{\infty}^{\prime}\right)$;

$\left(c_{10}\right)$ if $p \notin\left(\cup_{s_{i} \in \operatorname{Crit}_{\text {analytic }} \bar{H}} U_{s_{i}}^{\prime}\right) \cup U_{\infty}^{\prime}$, then $U_{p} \cap\left(\left(\cup_{s_{i} \in \operatorname{Crit}_{\text {analytic }} \bar{H}} U_{s_{i}}\right) \cup\right.$ $\left.U_{\infty}\right)=\emptyset$.

Let us add the neighbourhoods $U_{p}$ and $V_{p}$ to the sets $\left\{U_{s_{i}}\right\}$ and $\left\{V_{s_{i}}\right\}$ chosen above (here, one of $s_{i}=\infty$ ). As a result, we obtain two open coverings $\mathcal{V}=\left\{V_{p}\right\}$ and $\mathcal{U}=\left\{U_{p}\right\}$ of $\mathbb{C P}^{2}$.

For $U^{\prime}=U_{\infty}^{\prime}$ let $\left(w_{3, \infty}, \ldots, w_{m_{\infty}, \infty}\right)$ be functions in $\tilde{Y} \backslash \tilde{f}^{-1}\left(L_{\infty}\right)$ defined by $w_{j, \infty}=\tilde{f}^{*}\left(\rho_{\infty}\right) w_{j}^{\prime}$ for $j=3, \ldots, m_{\infty}$, where the functions $w_{j}^{\prime}$ were defined in (9).

For each $U_{s_{i}}^{\prime}$, where $s_{i} \in \operatorname{Crit}_{\text {analytic }} \bar{H}$, there are complex analytic functions $w_{1, s_{i}}^{\prime}, \ldots, w_{m_{s_{i}}, s_{i}}^{\prime}$ in $\widetilde{U}_{s_{i}}^{\prime}=\widetilde{f}^{-1}\left(U_{s_{i}}^{\prime}\right)$ such that these functions together with $\tilde{f}^{*}\left(u_{1}\right)$ and $\tilde{f}^{*}\left(v_{1}\right)$ give an analytic embedding of $\widetilde{U}_{s_{i}}^{\prime}$ to $\mathbb{C}^{m_{s_{i}}+2}$. Denote by $w_{j, s_{i}}=\widetilde{f}^{*}\left(\rho_{s_{i}}\right) w_{j, s_{i}}^{\prime}, 1 \leqslant j \leqslant m_{s_{i}}$, functions in $\widetilde{Y}$.

By construction of the open coverings, the preimage $\tilde{f}^{-1}\left(V_{p}\right)=$ $\coprod \widetilde{V}_{p, j}$ (resp. $\left.\tilde{f}^{-1}\left(U_{p}\right)=\coprod \widetilde{U}_{p, j}\right)$ splits into disjoint union of $m_{p}=n_{p}$ connected neighbourhoods $\widetilde{V}_{p, j}$ (resp. $\left.\widetilde{U}_{p, j}\right), j=1, \ldots, n_{p}$. If $p \in \bar{H}$, then a neighbourhood $\widetilde{U}_{p, j}$ is isomorphic to a subvariety of $\mathbb{C}^{3}$ given in coordinates $\left(u_{1}, v_{1}, w_{j, p}^{\prime}\right)$ by

$$
\left(w_{j, p}^{\prime}-w_{j, p}^{0}\right)^{r_{j, p}}=v_{1}-v_{1, p}\left(u_{1}\right)
$$

where $v_{1}-v_{1, p}\left(u_{1}\right)=0$ is the equation of $\bar{H}$ in $U_{p}$. Extend the functions $w_{j, p}^{\prime}$ by putting $w_{j, p \mid \widetilde{U}_{p, l}}^{\prime} \equiv 0$ for $l \neq j$ and choose constants $w_{j, p}^{0}, j=$ $1, \ldots, m_{p}$, so that the functions $\left(u_{1}, v_{1}, w_{1, p}^{\prime}, \ldots, w_{m_{p}, p}^{\prime}\right)$ define a smooth embedding of $\widetilde{U}_{p}$ to $\mathbb{C}^{m_{p}+2}$. Denote by $w_{j, p}=\widetilde{f}^{*}\left(\rho_{p}\right) w_{j, p}^{\prime}, j=1, \ldots, m_{p}$, functions in $\widetilde{Y}$.

Note that if $y \in \widetilde{f}^{-1}(\bar{H}) \cap \widetilde{V}_{p, j} \cap \widetilde{U}_{q, j}$, where $p, q \neq \infty$ and $p, q \notin$ Crit $_{\text {analytic }} \bar{H}$, then by the definition of the functions $w_{j, p}$ and $w_{j, q}$, we have $r_{j, p}=r_{j, q}=r_{j}$ and there is a $r_{j}$-th root $\zeta_{p, q}$ of unity such that

$$
\begin{array}{ll}
w_{j, q} & =\rho_{q}\left(u_{1}, v_{1}\right)\left(\zeta_{p, q}\left(w_{j, p}-w_{j, p}^{0}\right)+w_{j, p}^{0}\right) \\
w_{j^{\prime}, q} & \equiv 0 \quad \text { for } j^{\prime} \neq j
\end{array}
$$

in a neighbourhood of $y$. 
Similarly, if $y \in \widetilde{f}^{-1}(\bar{H}) \cap \widetilde{V}_{p, j} \cap \widetilde{U}_{q}$ (resp. if $y \in \widetilde{f}^{-1}(\bar{H}) \cap \widetilde{V}_{q} \cap \widetilde{U}_{p, j}$ ), where $q=\infty$ or $q \in \operatorname{Sing}_{\text {analytic }} \bar{H}$, then by the definition of the functions $w_{j, p}$ and $w_{i, q}$ and by properties $\left(c_{1}\right),\left(c_{4}\right),\left(c_{9}\right)$, and $\left(c_{10}\right)$, we have

$$
\left(w_{j, p}-w_{j, p}^{0}\right)^{r_{j}}=v_{1}-F\left(u_{1}\right)
$$

and

$$
w_{i, q}=\rho_{q}\left(u_{1}, v_{1}\right) h_{i}\left(u_{1}, v_{1}, w_{j, p}\right) \quad \text { for } 1 \leqslant i \leqslant m_{q}
$$

(resp. $w_{j, p}=\rho_{p}\left(u_{1}, v_{1}\right) h_{j}\left(u_{1}, v_{1}, w_{1, q}, w_{2, q}, w_{3, q}, \ldots, w_{m_{q}, q}\right)$, here $w_{1, q}$ and $w_{2, q}$ are constants if $q=\infty$ ) in a neighbourhood of $y$, where $F$ and all $h_{i}$ (resp. $h_{j}$ ) are analytic functions, and $v_{1}-F\left(u_{1}\right)=0$ is an analytic equation of some branch of $\bar{H}$.

If $p \notin \bar{H} \cup L_{\infty}$, then we can assume that $\tilde{f}$ defines an isomorphism of the neighbourhoods $\widetilde{U}_{p, j}$ and $U_{p}$ for $j=1, \ldots, m_{p}=N$. Choose $N$ different constants $w_{j, p}^{0}$ and define functions $w_{j, p}=\tilde{f}^{*}\left(\rho_{p}\right) w_{j, p}^{\prime}, j=$ $1, \ldots, m_{p}$, in $\tilde{Y}$, where the function $w_{j, p}^{\prime}$ is defined in $\widetilde{U}_{p}$ as follows

$$
w_{j, p}^{\prime}(q) \equiv \begin{cases}w_{j, p}^{0} & \text { if } q \in \widetilde{U}_{p, j} \\ 0 & \text { if } q \notin \widetilde{U}_{p, j} .\end{cases}
$$

Choose a finite covering $\widetilde{\mathcal{V}}_{0}=\left\{\widetilde{V}_{p_{i}, j} \mid 1 \leqslant i \leqslant k, 1 \leqslant j \leqslant m_{p_{i}}\right\} \cup\left\{\widetilde{V}_{\infty}\right\}$ of $\widetilde{Y}$, set

$$
M=m_{\infty}+\sum_{j=1}^{k} m_{p_{j}},
$$

$w_{3}=w_{3, \infty}, \ldots, w_{m_{\infty}}=w_{m_{\infty}, \infty}$, and enumerate the set of functions

$$
\left\{w_{j, p_{i}} \mid 1 \leqslant i \leqslant k, 1 \leqslant j \leqslant m_{p_{i}}\right\}
$$

by the numbers $m_{\infty}+1, \ldots, M$.

Consider a linear projection $p: \mathbb{C P}^{M} \rightarrow \mathbb{C P}^{2}$ given by

$$
\left(z_{0}: z_{1}: z_{2}: \cdots: z_{M}\right) \rightarrow\left(z_{0}: z_{1}: z_{2}\right) .
$$

The base locus of $p$ is a projective space $P \simeq \mathbb{C P}^{M-3}$ given by equations $z_{0}=z_{1}=z_{2}=0$. The restriction of $p$ to $\mathcal{L}=\mathbb{C P}^{M} \backslash P$ defines on $\mathcal{L}$ the structure of a vector bundle over $\mathbb{C P}^{2}$ the zero section of which is given by $z_{3}=\cdots=z_{M}=0$. Over the charts $\mathbb{C}_{i}^{2}$ with coordinates $\left(u_{i}, v_{i}\right)$, $i=1,2,3$, the bundle $\mathcal{L}$ is trivial, it is isomorphic to $\mathbb{C}_{i}^{M} \simeq \mathbb{C}_{i}^{2} \times \mathbb{C}_{i}^{M-2}$. In particular, $\left(z_{3} / z_{0}, \ldots, z_{M} / z_{0}\right)$ are coordinates in $\mathbb{C}_{1}^{M-2}$.

Over $\mathbb{C}_{1}^{2}$, consider a map $\alpha^{\prime}: \tilde{f}^{-1}\left(\mathbb{C}_{1}^{2}\right) \rightarrow \mathbb{C}_{1}^{M}$ given by

$$
\alpha^{\prime}(y)=\left(\tilde{f}^{*}\left(u_{1}\right)(y), \tilde{f}^{*}\left(v_{1}\right)(y), w_{3}(y), \ldots, w_{M}(y)\right) .
$$

Since for $p_{i} \neq \infty$ each $U_{p_{i}}$ is a subset of $\mathbb{C}_{1}^{2}$ and all functions $w_{j, p_{i}} \equiv 0$ at the points lying over the complement of $U_{p_{i}}$, the map $\alpha^{\prime}$ can be 
extended to a map $\alpha: \tilde{Y} \rightarrow \mathcal{L}$ as follows. Over the neighbourhood $\widetilde{f}^{-1}\left(V_{\infty}^{\prime}\right)$ the map $\alpha$ equals $i \circ h$, where $h$ was defined above and $i$ is a linear embedding of $\mathbb{C P}^{m_{\infty}}$ to $\mathbb{C P}^{M}$ given by

$$
i\left(\left(z_{0}: \ldots: z_{m_{\infty}}\right)\right)=\left(z_{0}: \ldots: z_{m_{\infty}}: 0: \ldots: 0\right) .
$$

It is easy to see that $\alpha$ is an embedding such that $\alpha\left(\widetilde{V}_{s_{i}}^{\prime}\right)$ and $\alpha\left(\widetilde{V}_{\infty}^{\prime}\right)$ are analytic subsets of $\mathcal{L}$ for $\widetilde{V}_{\infty}^{\prime}=\widetilde{f}^{-1}\left(V_{\infty}^{\prime}\right)$ and for all neighbourhoods $\widetilde{V}_{s_{i}}^{\prime}=\widetilde{f}^{-1}\left(V_{s_{i}}^{\prime}\right), s_{i} \in \operatorname{Crit}_{\text {analytic }} \bar{H}$.

Denote by $\Omega$ the restriction of the Fubini - Studi form $\Omega_{M}$ to $\mathcal{L}$. In the chart $\mathbb{C}_{1}^{M}$, it has the form

$$
\Omega=\frac{i \sum_{k=1}^{M}\left(d w_{k} \wedge d \bar{w}_{k}+\sum_{j \neq k}\left(\bar{w}_{j} w_{j} d w_{k} \wedge d \bar{w}_{k}-\bar{w}_{j} w_{k} d w_{j} \wedge d \bar{w}_{k}\right)\right)}{\left(1+\sum_{j=1}^{M} \bar{w}_{j} w_{j}\right)^{2}},
$$

where $w_{k}=\frac{z_{k}}{z_{0}}$ and $w_{1}=u_{1}, w_{2}=v_{1}$.

Denote by the same symbol $\bar{\varepsilon}=\left(\varepsilon_{1}, \varepsilon_{2}\right)$ a collection of two positive numbers and the linear transformation $\bar{\varepsilon}: \mathbb{C P}^{M} \rightarrow \mathbb{C P}^{M}$ given by

$$
\left(z_{0}: z_{1}: z_{2}: z_{3}: \ldots: z_{M}\right) \rightarrow\left(z_{0}: z_{1}: \varepsilon_{1} z_{2}: \varepsilon_{2} z_{3}: \ldots: \varepsilon_{2} z_{M}\right) .
$$

Denote by $\omega_{\bar{\varepsilon}}$ the restriction of the form $\Omega$ to $\widetilde{Y}_{\bar{\varepsilon}}=(\bar{\varepsilon} \circ \alpha)(\widetilde{Y})$. Let us show that there exist a positive constant $c_{1}$ and a positive function $c_{2}(t), t \in\left(0, c_{1}\right]$, such that $\widetilde{Y}_{\bar{\varepsilon}}$ is a symplectic in $\mathcal{L}$ for all $\bar{\varepsilon}=\left(\varepsilon_{1}, \varepsilon_{2}\right)$ with $\varepsilon_{1} \leqslant c_{1}, \varepsilon_{2} \leqslant c_{2}\left(\varepsilon_{1}\right)$. Note that if $\varepsilon_{1}$ is small enough, then the image of $\bar{H}$ under the map $\left(z_{0}: z_{1}: z_{2}\right) \rightarrow\left(z_{0}: z_{1}: \varepsilon_{1} z_{2}\right)$ becomes symplectic.

For each $\bar{\varepsilon}$, the form $\omega_{\bar{\varepsilon}}$ is a symplectic form at the points from the neighbourhoods $(\bar{\varepsilon} \circ \alpha)\left(\widetilde{V}_{s_{i}}^{\prime}\right)$, where $s_{i}$ is an analytic singular point of $\bar{H}$, and from $(\bar{\varepsilon} \circ \alpha)\left(\widetilde{V}_{\infty}^{\prime}\right)$, since $\widetilde{Y}_{\bar{\varepsilon}}$ is an analytic subvariety of $\mathcal{L}$ at these points.

For each point $y$ not belonging to the ramification locus of $\tilde{f}$, the variety $\widetilde{Y}$ is given locally at $\alpha(y)$ by equations $w_{j}=F_{j}\left(u_{1}, v_{1}\right), j=$ $3, \ldots, M$, where $F_{j}\left(u_{1}, v_{1}\right)$ are smooth functions in a neighbourhood of $\widetilde{f}(y)$. Therefore the variety $\widetilde{Y}_{\bar{\varepsilon}}$ is given locally at $(\bar{\varepsilon} \circ \alpha)(y)$ by equations $w_{j}=\varepsilon_{2} F_{j}\left(u_{1}, \frac{v_{1}}{\varepsilon_{1}}\right), j=3, \ldots, M$. It is easy to see that for each fixed $\varepsilon_{1}$ and for $\varepsilon_{2}$ being small enough, the form $\omega_{\bar{\varepsilon}}$ is symplectic at $(\bar{\varepsilon} \circ \alpha)(y)$, since the variety $\widetilde{Y}_{\bar{\varepsilon}}$ is very close to the algebraic variety given by $w_{j}=0$, $j=3, \ldots, M$, which is symplectic. 
Consider a point $y \in \widetilde{f}^{-1}(\bar{H})$ belonging to the ramification locus of $\widetilde{f}$ and such that $\widetilde{f}(y) \notin \widetilde{U}_{p}$ for $p \in \operatorname{Crit}_{\text {analytic }} \bar{H}$ and for $p=\infty$. By (10), renumbering the coordinates $w_{3}, \ldots, w_{M}$, we can assume that $\widetilde{Y}_{\bar{\varepsilon}}$ is given in a neighbourhood of $\alpha(y)$ by equations

$$
\begin{aligned}
\left(w_{3}-w_{3,0}\right)^{r} & =v_{1}-F\left(u_{1}\right), \\
w_{j} & =\rho_{j}\left(u_{1}, v_{1}\right) h_{j}\left(w_{3}\right), \quad j \geqslant 4,
\end{aligned}
$$

where $r \geqslant 2, \rho_{j}$ are smooth functions, $h_{j}=\zeta_{3, j}\left(w_{3}-w_{3,0}\right)+w_{j, 0}$ are analytic functions, $v_{1}-F\left(u_{1}\right)=0$ is the equation of a branch of $\bar{H}$ at the point $\tilde{f}(y)$, and the point $\alpha(y)=\left(u_{1,0}, F\left(u_{1,0}\right), w_{3,0}, \ldots, w_{M, 0}\right)$. Then the variety $\widetilde{Y}_{\bar{\varepsilon}}$ is given by equations

$$
\begin{aligned}
\varepsilon_{1}\left(w_{3}-\varepsilon_{2} w_{3,0}\right)^{r} & =\varepsilon_{2}^{r}\left(v_{1}-\varepsilon_{1} F\left(u_{1}\right)\right), \\
w_{j} & =\varepsilon_{2} \rho_{j}\left(u_{1}, \frac{v_{1}}{\varepsilon_{1}}\right) h_{j}\left(\frac{w_{3}}{\varepsilon_{2}}\right), \quad j \geqslant 4 .
\end{aligned}
$$

in a neighbourhood of $(\bar{\varepsilon} \circ \alpha)(y)=\left(u_{1,0}, \varepsilon_{1} F\left(u_{1,0}\right), \varepsilon_{2} w_{3,0}, \ldots, \varepsilon_{2} w_{M, 0}\right)$.

Denote by $A_{1}=\frac{\partial F}{\partial u_{1}}(\alpha(y)), A_{2}=\frac{\partial F}{\partial \bar{u}_{1}}(\alpha(y)), B_{j}=\frac{\partial h_{j}}{\partial w_{3}}(\alpha(y)), C_{j, 1}=$ $\frac{\partial \rho_{j}}{\partial u_{1}}(\alpha(y)), C_{j, 2}=\frac{\partial \rho_{j}}{\partial \bar{u}_{1}}(\alpha(y)), D_{j, 1}=\frac{\partial \rho_{j}}{\partial v_{1}}(\alpha(y)), D_{j, 2}=\frac{\rho_{j}}{\partial \bar{v}_{1}}(\alpha(y)), \rho_{j, 0}=$ $\rho_{j}(\alpha(y)), h_{j, 0}=h_{j}\left(w_{3,0}\right), j=4, \ldots, M$. It follows from (15) that at the point $(\bar{\varepsilon} \circ \alpha)(y)$, we have

$$
\begin{aligned}
& d v_{1}=\varepsilon_{1}\left(A_{1} d u_{1}+A_{2} d \bar{u}_{1}\right) \\
& d \bar{v}_{1}=\varepsilon_{1}\left(\bar{A}_{2} d u_{1}+\bar{A}_{1} d \bar{u}_{1}\right)
\end{aligned}
$$

and

$$
\begin{aligned}
& d w_{j}=\rho_{j, 0} B_{j} d w_{3}+\varepsilon_{2} h_{j, 0}\left(C_{j, 1} d u_{1}+C_{j, 2} d \bar{u}_{1}+D_{j, 1} \frac{d v_{1}}{\varepsilon_{1}}+D_{j, 2} \frac{d \bar{v}_{1}}{\varepsilon_{1}}\right) \\
& d \bar{w}_{j}=\rho_{j, 0} \bar{B}_{j} d \bar{w}_{3}+\varepsilon_{2} \bar{h}_{j, 0}\left(\bar{C}_{j, 2} d \bar{u}_{1}+\bar{C}_{j, 1} d u_{1}+\bar{D}_{j, 2} \frac{d v_{1}}{\varepsilon_{1}}+\bar{D}_{j, 1} \frac{d \bar{v}_{1}}{\varepsilon_{1}}\right)
\end{aligned}
$$$$
j=4, \ldots, M \text {. }
$$

If we substitute (16) to (17), we obtain that

$$
\begin{aligned}
d w_{j} & =\rho_{j, 0} B_{j} d w_{3}+\varepsilon_{2} \nu_{j} \\
d \bar{w}_{j} & =\rho_{j, 0} \bar{B}_{j} d \bar{w}_{3}+\varepsilon_{2} \bar{\nu}_{j},
\end{aligned}
$$

where the forms $\nu_{j}$ and $\bar{\nu}_{j}$ do not depend on $\bar{\varepsilon}$ for $j=4, \ldots, M$.

It follows from (16) and (18) that for each very small $\bar{\varepsilon}$ the tangent space of $\widetilde{Y}_{\bar{\varepsilon}}$ at the point $(\bar{\varepsilon} \circ \alpha)(y)$ is very close to the tangent space at the point $(\bar{\varepsilon} \circ \alpha)(y)$ of a linear algebraic variety $Z$ given by $v_{1}=\varepsilon_{1} F\left(u_{1,0}\right)$, $w_{j}-w_{j, 0}=\rho_{j, 0} B_{j}\left(w_{3}-w_{3,0}\right), j=4, \ldots, M$. Therefore for each very small $\bar{\varepsilon}$ the form $\omega_{\bar{\varepsilon}}$ is symplectic at $(\bar{\varepsilon} \circ \alpha)(y)$. By continuity, it is symplectic in some neighbourhood of $(\bar{\varepsilon} \circ \alpha)(y)$.

Consider a point $y \in \widetilde{f}^{-1}(\bar{H})$ belonging to the ramification locus of $\tilde{f}$ and such that $\tilde{f}(y) \in U_{p}$ for some $p \in \operatorname{Crit}_{\text {analytic }} \bar{H}$ or $p=\infty$. By (11) 
and (12), renumbering the coordinates $w_{3}, \ldots, w_{M}$, we can assume that there is some $n, 3 \leqslant n \leqslant M$, such that $\widetilde{Y}_{\bar{\varepsilon}}$ is given in a neighbourhood of $\alpha(y)$ by equations

$$
\begin{aligned}
h_{j}\left(u_{1}, v_{1}, w_{3}, \ldots, w_{n}\right) & =0, \quad j=3, \ldots, n \\
\rho_{j}\left(u_{1}, v_{1}\right) h_{j}\left(u_{1}, v_{1}, w_{3}, \ldots, w_{n}\right) & =w_{j}, \quad j=n+1 \ldots, M,
\end{aligned}
$$

where $\rho_{j}$ are smooth functions and $h_{j}$ are analytic functions at the point $\widetilde{f}(y)$. Let $\left(u_{1,0}, v_{1,0}, w_{3,0}, \ldots, w_{M, 0}\right)$ be the coordinates of the point $\alpha(y)$. Then the variety $\widetilde{Y}_{\bar{\varepsilon}}$ is given in a neighbourhood of $(\bar{\varepsilon} \circ \alpha)(y)=$ $\left(u_{1,0}, \varepsilon_{1} v_{1,0}, \varepsilon_{2} w_{3,0}, \ldots, \varepsilon_{2} w_{M, 0}\right)$ by equations

$$
\begin{aligned}
h_{j}\left(u_{1}, \frac{v_{1}}{\varepsilon_{1}}, \frac{w_{3}}{\varepsilon_{2}}, \ldots, \frac{w_{n}}{\varepsilon_{2}}\right) & =0, \quad j=3, \ldots, n \\
\varepsilon_{2} \rho_{j}\left(u_{1}, \frac{v_{1}}{\varepsilon_{1}}\right) h_{j}\left(u_{1}, \frac{v_{1}}{\varepsilon_{1}}, \frac{w_{3}}{\varepsilon_{2}}, \ldots, \frac{w_{n}}{\varepsilon_{2}}\right) & =w_{j}, \quad j=n+1 \ldots, M .
\end{aligned}
$$

Set $A_{j, l}=\frac{\partial h_{j}}{\partial w_{l}}(\alpha(y))$ for $j \leqslant j \leqslant M, 1 \leqslant l \leqslant n$, (here $w_{1}=u_{1}$ and $\left.w_{2}=v_{1}\right)$ and by $B_{j}=\rho_{j}\left(u_{1,0}, v_{1,0}\right)$ for $n+1 \leqslant j \leqslant M$.

It follows from (20) that for each fixed positive $\varepsilon_{1}$ and for very small $\varepsilon_{2}$ the tangent space of $\widetilde{Y}_{\bar{\varepsilon}}$ at the point $(\bar{\varepsilon} \circ \alpha)(y)$ is very close to the tangent space at the point $(\bar{\varepsilon} \circ \alpha)(y)$ of a linear algebraic variety $Z$ given by

$$
\begin{aligned}
& \sum_{l=3}^{n} A_{j, l}\left(w_{l}-\varepsilon_{2} w_{l, 0}\right)=-A_{j, 1}\left(u_{1}-u_{1,0}\right)-\frac{A_{j, 2}}{\varepsilon_{1}}\left(v_{1}-\varepsilon_{1} v_{1,0}\right), 3 \leqslant j \leqslant n \\
& B_{j} \sum_{l=3}^{n} A_{j, l}\left(w_{l}-\varepsilon_{2} w_{l, 0}\right)=w_{j}-\varepsilon_{2} w_{j, 0}, \quad n+1 \leqslant j \leqslant M .
\end{aligned}
$$

Therefore for fixed $\varepsilon_{1}$ and for each very small $\varepsilon_{2}$, the form $\omega_{\bar{\varepsilon}}$ is symplectic at $(\bar{\varepsilon} \circ \alpha)(y)$. By continuity, it is symplectic in some neighbourhood of $(\bar{\varepsilon} \circ \alpha)(y)$.

To complete the proof, it suffices to apply the compactness of $\widetilde{Y}$.

By Lemma 2.1 and by Hironaka's Theorem on resolution of singularities, we have

Corollary 3.2. Let $\widetilde{f}: \widetilde{Y} \rightarrow \mathbb{C P}^{2}$ be a finite covering branched along a Hurwitz curve $\bar{H}$ (possibly, with negative nodes) and, maybe, along $L_{\infty}$, and associated with a monodromy $\mu: \pi_{1}\left(\mathbb{C}^{2} \backslash H\right) \rightarrow \Sigma_{N}$. Then there exist collections of positive integers $\left(M_{1}, \ldots, M_{k}\right)$ and $\left(n_{1}, \ldots, n_{k}\right)$ such that a resolution $\bar{Y}$ of singularities of $\tilde{Y}$ can be embedded as a symplectic submanifold to $\left(\mathbb{C P}^{M_{1}} \times \cdots \times \mathbb{C P}^{M_{k}}, \Omega_{n_{1}, \ldots, n_{k}}\right)$, where $\Omega_{n_{1}, \ldots, n_{k}}=$ $n_{1} p_{1}^{*}\left(\Omega_{M_{1}}\right)+\cdots+n_{k} p_{k}^{*}\left(\Omega_{M_{k}}\right)$ and $\Omega_{M_{j}}$ is the Fubini - Studi symplectic form on $\mathbb{C P}^{M_{j}}$. 
Theorem 3.3. In the notations of the proof of Theorem 3.1 , let $\tilde{Y}$ be a smooth manifold. Then the symplectic structure, constructed in the proof of Theorem 3.1 and given by the symplectic form $\omega_{\bar{\varepsilon}}$, does neither depend on $\bar{\varepsilon}$ if the coordinates of $\bar{\varepsilon}$ are small enough nor on the choice the coverings $\mathcal{U}$ and $\mathcal{V}$, and on the choice of the functions $w_{i, j}$.

Moreover, if $i: \widetilde{Y} \hookrightarrow \mathbb{C P}^{N}$ is an algebraic embedding (in the case when $\bar{H}$ is an algebraic curve) and $\widetilde{f}=p \circ i$, where $p: \mathbb{C P}^{N} \rightarrow \mathbb{C P}^{2}$ is a linear projection, then $\left(\widetilde{Y}, \omega_{\bar{\varepsilon}}\right)$ and $\left(\widetilde{Y}, i^{*}\left(\Omega_{N}\right)\right)$ are symplectomorphic for $\bar{\varepsilon}$ being small enough, where $\Omega_{N}$ is the Fubini - Studi form on $\mathbb{C P}^{N}$ and $\omega_{\bar{\varepsilon}}$ is the form constructed in the proof of Theorem 3.1 .

Proof. Let $\omega_{\bar{\varepsilon}}=(\bar{\varepsilon} \circ \alpha)^{*}(\Omega)$, where the embedding $\alpha: \tilde{Y} \rightarrow \mathcal{L}$ was constructed in the proof of Theorem 3.1. Note that for each $\bar{\varepsilon}, 0<$ $\varepsilon_{1} \leqslant c_{1}$ and $0<\varepsilon_{2} \leqslant c_{2}\left(\varepsilon_{1}\right)$, the class $\left[\omega_{\bar{\varepsilon}}\right] \in H^{2}(\widetilde{Y}, \mathbb{Z})$ is dual to the class $\left[\widetilde{f}^{-1}(L)\right] \in H_{2}(\widetilde{Y}, \mathbb{Z})$, where $L$ is a line in $\mathbb{C P}^{2}$. Therefore, by Moser's stability theorem for symplectic structures (see [16], Theorem $3.17)$, the forms $\omega_{\bar{\varepsilon}}$ define the same symplectic structure if $0<\varepsilon_{1} \leqslant c_{1}$ and $0<\varepsilon_{2} \leqslant c_{2}\left(\varepsilon_{1}\right)$.

The symplectic structure on $\widetilde{Y}$, defined by the forms $\omega_{\bar{\varepsilon}}$ does not depend on the choice of the coverings $\left\{U_{i}\right\}$ and $\left\{V_{i}\right\}$, and the choice of the functions $w_{i, j}$ defining the embedding $\alpha$. Indeed, let two collections $\left\{w_{i, j}^{\prime}\right\}$ and $\left\{w_{i, j}^{\prime \prime}\right\}$ of functions define two embeddings $\alpha^{\prime}: \widetilde{Y} \rightarrow \mathcal{L}^{\prime} \subset$ $\mathbb{C P}^{M^{\prime}}$ and $\alpha^{\prime \prime}: \widetilde{Y} \rightarrow \mathcal{L}^{\prime \prime} \subset \mathbb{C P}^{M^{\prime \prime}}$, and put $\omega_{\bar{\varepsilon}}^{\prime}=\left(\bar{\varepsilon} \circ \alpha^{\prime}\right)^{*}\left(\Omega^{\prime}\right)$ and $\omega_{\bar{\varepsilon}}^{\prime \prime}=\left(\bar{\varepsilon} \circ \alpha^{\prime \prime}\right)^{*}\left(\Omega^{\prime \prime}\right)$, where $\Omega^{\prime}$ and $\Omega^{\prime \prime}$ are the Fubini - Studi symplectic forms on $\mathbb{C P}^{M^{\prime}}$ and $\mathbb{C P}^{M^{\prime \prime}}$ respectively. Then we have an embedding $\alpha^{\prime} \times \alpha^{\prime \prime}: \widetilde{Y} \rightarrow \mathcal{L}^{\prime} \times_{\mathbb{C P}^{2}} \mathcal{L}^{\prime \prime}$. Note that the form $\Omega_{t}=t\left(p^{\prime}\right)^{*}\left(\Omega^{\prime}\right)+(1-$ $t)\left(p^{\prime \prime}\right)^{*}\left(\Omega^{\prime \prime}\right)$ is a Kähler form for each $t \in[0,1]$. Since the segment $[0,1]$ is compact, applying a similar calculation as in the proof of Theorem [3.1. one can show that there exists $\bar{\varepsilon}=\bar{\varepsilon}^{\prime}=\bar{\varepsilon}^{\prime \prime}, 0<\varepsilon_{1} \leqslant \min \left(c_{1}^{\prime}, c_{1}^{\prime \prime}\right)$, $0<\varepsilon_{2} \leqslant \min \left(c_{2}^{\prime}\left(\varepsilon_{1}\right), c_{2}^{\prime \prime}\left(\varepsilon_{1}\right)\right)$, such that $\omega_{t, \bar{\varepsilon}}=\left(\left(\bar{\varepsilon} \circ\left(\alpha^{\prime} \times \alpha^{\prime \prime}\right)\right)^{*}\left(\Omega_{t}\right)\right.$ is a symplectic form on $\tilde{Y}$ for all $t \in[0,1]$. On the other hand, $\omega_{0, \bar{\varepsilon}}=\omega_{\bar{\varepsilon}}^{\prime}$ and $\omega_{1, \bar{\varepsilon}}=\omega_{\bar{\varepsilon}}^{\prime \prime}$, and the forms $\omega_{t, \bar{\varepsilon}}$ belong to the same cohomology class. Therefore, by Moser's stability theorem for symplectic structures, the forms $\omega_{t, \bar{\varepsilon}}$ define the same symplectic structure on $\tilde{Y}$.

In the case of an algebraic embedding $i: \tilde{Y} \hookrightarrow \mathbb{C P}^{N}$ such that $\widetilde{f}=p \circ i$, where $p: \mathbb{C P}^{N} \rightarrow \mathbb{C P}^{2}$ is a linear projection, denote by $\alpha^{\prime}$ : $\widetilde{Y} \rightarrow \mathcal{L}^{\prime} \subset \mathbb{C P}^{M^{\prime}}$ any embedding constructed in the proof of Theorem 3.1 and put $\alpha^{\prime \prime}=i$. Applying the same arguments as above, it is easy to see that $\left(\tilde{Y}, \omega_{\bar{\varepsilon}}\right)$ and $\left(\widetilde{Y}, i^{*}\left(\Omega_{N}\right)\right)$ are symplectomorphic for $\bar{\varepsilon}$ being small 
enough, since the symplectic manifolds $\left(\widetilde{Y}, \omega_{\bar{\varepsilon}}^{\prime \prime}\right)$ are symplectomorphic for all positive collections $\bar{\varepsilon}$, where $\omega_{\bar{\varepsilon}}^{\prime \prime}=\left(\bar{\varepsilon} \circ \alpha^{\prime \prime}\right)^{*}\left(\Omega_{N}\right)$.

\section{EMbedDings OF CYCLIC COVERINGS OF THE PLANE INTO RATIONAL PROJECTIVE 3-FOLDS}

In this section, we use the notations and assumptions of section 1]

Let $\bar{H}$ be a Hurwitz curve of degree $m$. Consider the infinite cyclic covering $f=f_{\infty}: X_{\infty} \rightarrow X^{\prime}=\mathbb{C}^{2} \backslash H$ corresponding to the epimorphism $\nu: \pi_{1}\left(\mathbb{C}^{2} \backslash H\right) \rightarrow \mathbb{F}_{1}$. The covering $f_{\infty}$ can be factorized through the cyclic covering $f_{n}: X_{n}^{\prime} \rightarrow \mathbb{C}^{2} \backslash H$ associated with the epimorphism $\bmod _{n} \circ \nu, f_{\infty}=g_{n} \circ f_{n}$.

In this section, we will show that the covering $f_{n}$ can be extended to a smooth map $\bar{f}_{n}: \bar{X}_{n} \rightarrow \mathbb{C P}^{2}$ branched along $\bar{H}$ and, maybe, along $L_{\infty}$ (if $n$ is not a divisor of $\operatorname{deg} \bar{H}$, then $\bar{f}_{n}$ is branched along $L_{\infty}$ ), where $\bar{X}_{n}$ is a real smooth 4 -fold.

Theorem 4.1. A resolution of singularities $\bar{X}_{n}$ of a cyclic covering of $\mathbb{C P}^{2}$ of degree $n$, branched along a Hurwitz curve $\bar{H}$ and, maybe, along $L_{\infty}$, can be embedded into some rational projective 3-fold (equipped with integer Kähler symplectic structure) as a symplectic submanifold.

Proof. Since $\mathbb{C}^{2} \backslash H_{1}$ and $\mathbb{C}^{2} \backslash H_{2}$ are diffeomorphic for $H$-isotopic Hurwitz curves $\bar{H}_{1}$ and $\bar{H}_{2}$, we can assume that $\bar{H}$ satisfies conditions (6) and (8).

By Lemma 2.1 and by Hironaka's Theorem on resolution of singularities, it suffices to show that for some extension $\tilde{f}_{n}: \widetilde{X}_{n} \rightarrow \mathbb{C P}^{2}$ of $f_{n}^{\prime}: X_{n}^{\prime} \rightarrow X^{\prime}$, the variety $\widetilde{X}_{n}$ can be imbedded to some rational projective 3-fold (equipped with integer Kähler symplectic structure) as a symplectic subvariety with analytic singularities.

To show this, denote by $d$ the smallest non-negative integer for which $m+d$ is divisible by $n$. Put $m+d=k n$ and consider the line bundle $p: \mathcal{L}(k) \rightarrow \mathbb{C P}^{2}$ (see section 1) associated with the sheaf $\mathcal{O}_{\mathbb{C P}^{2}}(k)$. By Lemma 1.4, the Hurwitz curve $\bar{H}$ coincides with the zero locus of a smooth section $\bar{s}_{m}$ of $\mathcal{L}(m)$ over $\mathbb{C P}^{2}$ such that $\bar{s}_{m}$ is analytic in a neighbourhood $U$ of $L_{\infty}$ and in neighbourhoods of all critical points of $\bar{H}$.

Denote by $\bar{s}_{d}$ the section of $\mathcal{L}(d)$ defined over $\mathbb{C}_{2}^{2}$ by $w_{2}=u_{2}^{d}$. The product

$$
\bar{s}_{m+d}=\bar{s}_{m} \bar{s}_{d}
$$

is a section of $\mathcal{L}(m+d)$, where $\bar{s}_{m}$ is a section of $\mathcal{L}(m)$ satisfying all conditions from Lemma 1.4. 
Define $\alpha: \widetilde{X}_{n} \hookrightarrow \mathcal{L}(k)$ by the equation

$$
w_{i}^{n}=\bar{s}_{m+d}\left(u_{i}, v_{i}\right)
$$

and put $\tilde{f}_{n}=p_{\mid X_{n}}$, where $p: \mathcal{L}(k) \rightarrow \mathbb{C P}^{2}$ is the morphism defining on $\mathcal{L}(k)$ the structure of the line bundle. In particular, $\widetilde{X}_{n}$ is given by the equation

$$
w_{1}^{n}=F_{1}\left(u_{1}, v_{1}\right)
$$

in $\mathbb{C}_{1}^{3}$ and it is given by

$$
w_{2}^{n}=u_{2}^{d} F_{2}\left(u_{2}, v_{2}\right)
$$

in $\mathbb{C}_{2}^{3}$.

Obviously, over $\mathbb{C P}^{2} \backslash\left(\bar{H} \cup L_{\infty}\right)$ the covering $\tilde{f}_{n}$ is an unramified $n$-sheeted cyclic covering. Next, all singular points of the variety $\widetilde{X}_{n}$ lie over singular points of $\bar{H}$ and, maybe, over $L_{\infty}$. Moreover, by construction of the section $\bar{s}_{m+d}$, the set $\operatorname{Sing} \widetilde{X}_{n}$ is complex analytic in some neighbourhood $U \subset \mathcal{L}(k)$.

The line bundle $\mathcal{L}(k)$ is a quasi-projective variety and it can be compactified to a projective three dimensional rational manifold $\overline{\mathcal{L}}(k)$ by adding a section "at infinity".

The variety $\overline{\mathcal{L}}(k)$ has many different embeddings to projective spaces, since its Picard group $\operatorname{Pic}(\overline{\mathcal{L}}(k)) \simeq \mathbb{Z} \oplus \mathbb{Z}$. We can choose one of these embeddings, for example, the following one.

In the neighbourhood $\mathbb{C}_{1}^{3}$ with coordinates $\left(u_{1}, v_{1}, w_{1}\right)$ consider monomials $u_{1}^{a_{1}} v_{1}^{a_{2}} w_{1}^{a_{3}}, 0 \leqslant a_{1}+a_{2}+k a_{3} \leqslant k+1$, the number of which is equal to $\frac{(k+2)(k+3)}{2}+3$. Put $N=\frac{(k+2)(k+3)}{2}+2$ and consider a rational map $h: \overline{\mathcal{L}}(k) \rightarrow \mathbb{C P}^{N}$ given in $\mathbb{C}_{1}^{3}$ by $z_{\bar{a}}=u_{1}^{a_{1}} v_{1}^{a_{2}} w_{1}^{a_{3}}$, where $\bar{a}=\left(a_{1}, a_{2}, a_{3}\right)$ are triples of integers and $z_{\bar{a}}$ are homogeneous coordinates in $\mathbb{C P}^{N}$. It is easily to check that $h$ is an embedding.

Consider the Fubini - Studi form $\Omega_{N}$ on $\mathbb{C P}^{N}$ and denote by $\Omega=$ $h^{*}\left(\Omega_{N}\right)$ its pull back.

As in the proof of Theorem [3.1 denote by the same symbol $\bar{\varepsilon}=$ $\left(\varepsilon_{1}, \varepsilon_{2}\right)$ a collection of two positive numbers and automorphism of $\overline{\mathcal{L}}(k)$ given in $\mathbb{C}_{1}^{3}$ by $\left(u_{1}, v_{1}, w_{1}\right) \rightarrow\left(u_{1}, \varepsilon_{1} v_{1}, \varepsilon_{2} w_{1}\right)$.

The calculations (we omit them) similar to the calculations done in the proof of Theorem 3.1 show that there exist a positive constant $c_{1}$ and a positive function $c_{2}(t)$ such that $\widetilde{X}_{\bar{\varepsilon}}=(\bar{\varepsilon} \circ \alpha)\left(X_{n}\right)$ is a sympectic subvariety of $\mathcal{L}$ with analytic singularities for all $\bar{\varepsilon}=\left(\varepsilon_{1}, \varepsilon_{2}\right)$ with $\varepsilon_{1} \leqslant c_{1}, \varepsilon_{2} \leqslant c_{2}\left(\varepsilon_{1}\right)$ 


\section{Alexander polynomials of Hurwitz $C$-groups}

Let $\bar{H}$ be a Hurwitz (resp. topological Hurwitz) curve of degree $m$. Since any Zariski - van Kampen presentation of $\pi_{1}\left(\mathbb{C}^{2} \backslash H\right)$ is a $C$ presentation of a Hurwitz $C$-group of degree $m$, Theorems 0.1 and 0.2 are corollaries of the following Theorems 5.1 and 5.2 .

Theorem 5.1. Let $G \in \mathcal{H}$ be a Hurwitz $C$-group of degree $m$ and $\Delta(t)$ its Alexander polynomial. Then

(i) $\Delta(t) \in \mathbb{Z}[t]$

(ii) $\Delta(0)= \pm 1$;

(iii) the roots of $\Delta(t)$ are $m$-th roots of unity;

(iv) the rank of the free part of $N^{\prime} / N^{\prime \prime}$ is equal to $\operatorname{deg} \Delta(t)$;

$(v)$ the action of $h_{\mathbb{C}}$ on $N / N^{\prime} \otimes \mathbb{C}$ is semisimple.

Proof. Consider the exact sequence of groups

$$
1 \rightarrow N \rightarrow G \stackrel{\nu}{\longrightarrow} \mathbb{F}_{1} \rightarrow 1 \text {. }
$$

This exact sequence induces an automorphism $\widetilde{h} \in$ Aut $N$ (an action of the $C$-generator $x \in \mathbb{F}_{1}$ on $\left.N\right)$ given by $\widetilde{h}(n)=\widetilde{x}^{-1} n \widetilde{x}$ for $n \in N$, where $\widetilde{x}$ is one of the $C$-generators of $G$. Evidently, the automorphism $\widetilde{h}$ is defined uniquely up to inner automorphisms of the group $N$, therefore $\widetilde{h}$ defines an automorphism $h \in \operatorname{Aut} N / N^{\prime}$.

In 13, it was proved that for a Hurwitz $C$-group $G$, the group $N$ is finitely presented. Therefore $N / N^{\prime}$ is a finitely generated abelian group. Let $N / N^{\prime}=T \oplus F$ be a decomposition into the direct sum of the torsion subgroup $T$ and a free abelian group $F$. Note that $T$ is a finite group and $F$ is finitely generated. The automorphism $h$ of $N / N^{\prime}$ induces an automorphism of $T$ and therefore an automorphism $\bar{h}$ of $F \simeq\left(N / N^{\prime}\right) / T$. If one chooses a free basis of the $\mathbb{Z}$-module $F$ over $\mathbb{Z}$, then this automorphism will be given by a matrix $H$ with integer coefficients. Since the automorphism $h_{\mathbb{C}}$ of $N / N^{\prime} \otimes \mathbb{C}$ can be given by the same matrix $H$, the polynomial $\Delta(t)=\operatorname{det}(H-t \mathrm{Id}) \in \mathbb{Z}[t]$. And since $\bar{h} \in \operatorname{Aut} F$, then $\operatorname{det} \widetilde{H}= \pm 1$ and therefore, $\Delta(0)= \pm 1$.

Let us show that $\widetilde{h}^{m}$ is an inner automorphism of $N$. Indeed, since $G$ is a Hurwitz group of degree $m$, it is generated by $C$-generators $x_{1}, \ldots, x_{m}$ such that the product $x_{1} \ldots x_{m}$ belongs to the center of $G$. Therefore the element $\widetilde{x}^{m}=\widetilde{n} \cdot x_{1} \ldots x_{m}$, where $\widetilde{n} \in N$, induces on $N$ an inner automorphism. Thus the induced automorphisms $h^{m}$ of $N / N^{\prime}$ and $h_{\mathbb{C}}^{m}$ of $N / N^{\prime} \otimes \mathbb{C}$ are trivial, that is, $h_{\mathbb{C}}^{m}=\mathrm{Id}$.

Since $h_{\mathbb{C}}^{m}=\mathrm{Id}$, all roots of the polynomial $\Delta(t)=\operatorname{det}\left(h_{\mathbb{C}}-t \mathrm{Id}\right)$ are $m$-th roots of unity, and the action of $h_{\mathbb{C}}$ on $N / N^{\prime} \otimes \mathbb{C}$ is semisimple. 
Theorem 5.2. Let $G$ be an irreducible Hurwitz $C$-group, then

(i) $\Delta(1)=1$;

(ii) $\operatorname{deg} \Delta(t)$ is an even number;

(iii) $\Delta(t)$ is a reciprocal polynomial.

Proof. It follows from Lemma 6 in [10] that $\Delta(1)= \pm 1$. Let us show that $t=-1$ also is not a root of $\Delta(t)$. Indeed, if $t=-1$ is a root of $\Delta(t)$, then $\Delta(t)=(t+1) P(t)$, where $P(t)$ is a polynomial with integer coefficients. Therefore $2 P(1)= \pm 1$. But this is impossible, since $P(1)$ is an integer.

By Theorem 5.1, all roots of $\Delta(t) \in \mathbb{Z}[t]$ are roots of unity. They are non-real, since $t= \pm 1$ are not roots of $\Delta(t)$. Thus, deg $\Delta(t)$ is an even number and $(i i)$ is proved.

It is well known that if $\lambda$ is a primitive $k$-th root of unity, $k>2$, and a polynomial $P(t) \in \mathbb{Z}[t]$ has $\lambda$ as one of its roots, then all primitive $k$-th roots of unity are roots of $P(t)$. In particular, $\lambda^{-1}$ is also a root of $P(t)$ and $(i i i)$ is proved.

Since $\Delta(t)=\operatorname{det}\left(h_{\mathbb{C}}-t \mathrm{Id}\right)$ and $\operatorname{deg} \Delta(t)$ is an even number, then $\Delta(t)=t^{\operatorname{deg} \Delta(t)}+\ldots$. Let $\Delta(t)=\prod_{i} \Phi_{k_{i}}(t)$ be a factorization as the product of $k_{i}$-th cyclotomic polynomials. Now statement $(i)$ follows from well-known Lemma 5.3, since $\Delta(1)= \pm 1$.

Lemma 5.3. Let $\Phi_{k}(t)$ be a $k$-th cyclotomic polynomial, $k>1$. Then

$$
\Phi_{k}(1)= \begin{cases}p & \text { if } k=p^{n} \text { for some prime number } p \\ 1 & \text { if } k \neq p^{n} \text { for any prime number } p .\end{cases}
$$

Proof. By induction on $k>1$, Lemma 5.3 follows from the equalities

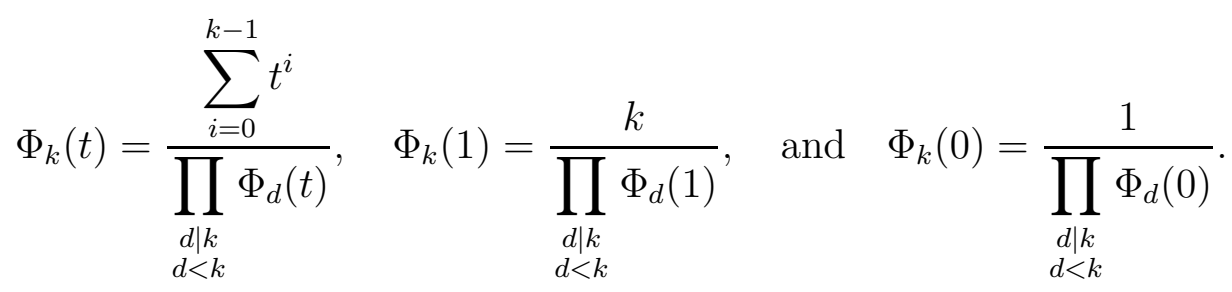

Corollary 0.3 is a consequence of

Corollary 5.4. Let $G$ be an irreducible Hurwitz $C$-group of degree $m=p^{n}$, where $p$ is a prime number. Then

(i) $\Delta(t) \equiv 1$;

(ii) the group $G^{\prime} / G^{\prime \prime}$ is a finite abelian group. 
Proof. By Theorem 5.1, all roots of $\Delta(t)$ are $m$-th roots of unity. Let $\lambda$ be one of the roots. Assume that $\lambda$ is a primitive $p^{k}$-th root of unity, $1 \leqslant k \leqslant n$. Then $\lambda$ is a root of the $p^{k}$-th cyclotomic polynomial

$$
\Phi_{p^{k}}(t)=\sum_{i=0}^{p-1} t^{i p^{k-1}}
$$

and there is a polynomial $f(t) \in \mathbb{Z}[t]$ such that $\Delta(t)=\Phi_{p^{k}}(t) f(t)$. By Theorem 5.2. $\Phi_{p^{k}}(1) f(1)= \pm 1$. On the other hand, $f(1) \in \mathbb{Z}$ and $\Phi_{p^{k}}(1)=p$. Therefore $\Delta(t)$ has no roots. Thus, $\operatorname{deg} \Delta(t)=0$ and the group $G^{\prime} / G^{\prime \prime}$ has no free part, that is, it is a finite abelian group.

Lemma 5.5. Let $G_{1}$ and $G_{2}$ be $C$-groups and $\Delta_{1}(t)$ and $\Delta_{2}(t)$ their Alexander polynomials. Assume that there is a $C$-epimorphism $f$ : $G_{1} \rightarrow G_{2}$. Then $\Delta_{2}(t)$ is a divisor of $\Delta_{1}(t)$.

Proof. Denote by $N_{i}$ the kernel of the canonical $C$-epimorphism $\nu_{i}$ : $G_{i} \rightarrow \mathbb{F}_{1}, i=1,2$. It is easy to see that the homomorphism $g$ in the commutative diagram

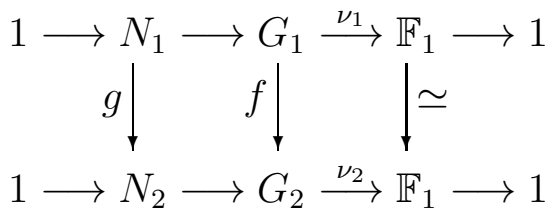

is an epimorphism. This diagram induces the following commutative diagram

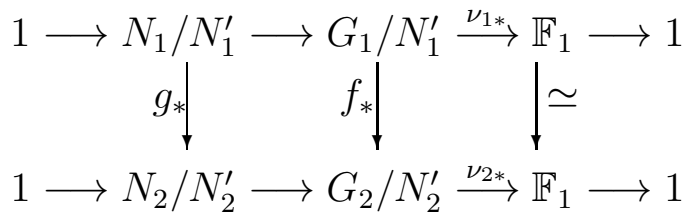

in which $g_{*}$ is also an epimorphism.

It follows from diagram $(*)$ that $\Delta_{2}(t)$ is a divisor of $\Delta_{1}(t)$, since $h_{2}\left(g_{*}(n)\right)=g_{*}\left(h_{1}(n)\right)$ for any $n \in N_{1} / N_{1}^{\prime}$.

Theorem 5.6. Let $G$ be a Hurwitz $C$-group of degree $m$. Then its Alexander polynomial $\Delta(t)$ divides the polynomial $(t-1)\left(t^{m}-1\right)^{m-2}$.

Proof. Consider the Hurwitz $C$-group

$$
\widetilde{G}_{m}=<x_{1}, \ldots, x_{m} \mid\left[x_{i}, x_{1} \ldots x_{m}\right]=1, i=1, \ldots, m>.
$$

For any Hurwitz $C$-group $G$ of degree $m$ there is a natural $C$ epimorphism $f: \widetilde{G}_{m} \rightarrow G$ sending the $C$-generators $x_{i}$ of $\widetilde{G}_{m}$ to the 
$C$-generators $x_{i}$ of $G$ for which the product $x_{1} \ldots x_{m}$ belongs to the center of $G$. Therefore to prove Theorem [5.6, it is sufficient to show that the Alexander polynomial of $\widetilde{G}_{m}$ is equal to $(-1)^{m-1}(t-1)\left(t^{m}-1\right)^{m-2}$.

Denote by $\widetilde{N}_{m}$ the kernel of $\nu: \widetilde{G}_{m} \rightarrow \mathbb{F}_{1}$ and put $y=x_{1} \ldots x_{m}$. Without loss of generality, we can assume that $\widetilde{h}(n)=x_{m} n x_{m}^{-1}$ for $n \in \widetilde{N}_{m}$. In [13], applying the Reidemeister - Schreier method, it was shown that $\widetilde{N}_{m}$ is generated be the elements

$$
a_{k, j}=x_{m}^{k} x_{j} x_{m}^{-(k+1)},
$$

where $j=2, \ldots, m-1, k \in \mathbb{Z}$, and by the elements

$$
a_{k, m}=x_{m}^{k} y x_{m}^{-(k+m)},
$$

where $k \in \mathbb{Z}$. Then the action $\widetilde{h}$ is given by $\widetilde{h}\left(a_{k, j}\right)=a_{k+1, j}$.

The relations

$$
y x_{j}=x_{j} y, \quad j=2, \ldots, m,
$$

give rise (see [13]) to relations

$$
a_{k, m}=a_{k+1, m}
$$

for $k \in \mathbb{Z}$ and to relations

$$
a_{k, m} a_{k+m, j} a_{k+1, m}^{-1}=a_{k, j}
$$

for $j=2, \ldots, m-1$ and $k \in \mathbb{Z}$. Therefore $\widetilde{N}_{m}$ is a free group generated by $a_{0, m}$ and $a_{k, j}, k=1, \ldots, m, j=2, \ldots, m-1$.

We have $\widetilde{h}\left(a_{0, m}\right)=a_{0, m}$ and

$$
\widetilde{h}\left(a_{k, j}\right)=a_{k+1, j}
$$

for $k=1, \ldots, m-1, j=2, \ldots, m-1$ and

$$
\widetilde{h}\left(a_{m, j}\right)=a_{0, m}^{-1} a_{1, j} a_{0, m}
$$

for $j=2, \ldots, m-1$.

Let $\bar{a}_{k, j}$ be the image of $a_{k, j}$ in $\widetilde{N}_{m} / \widetilde{N}_{m}^{\prime}$. Then the action $h$ is given by

$$
\begin{array}{rlrl}
\widetilde{h}\left(\bar{a}_{0, m}\right) & =\bar{a}_{0, m}, & \\
h\left(\bar{a}_{k, j}\right) & =\bar{a}_{k+1, j} & & \text { for } k=1, \ldots, m-1, j=2, \ldots, m-1, \\
h\left(\bar{a}_{m, j}\right) & =\bar{a}_{1, j} \quad & \text { for } j=2, \ldots, m-1 .
\end{array}
$$

Simple computations show that the characteristic polynomial of $h$ is equal to $(-1)^{m-1}(t-1)\left(t^{m}-1\right)^{m-2}$. 
It is easy to check (10, Lemma 4$)$ that $G / G^{\prime}$ is a finitely generated free abelian group for any $C$-group $G$. Moreover, the canonical epimorphism ab : $G \rightarrow G / G^{\prime}$ is a $C$-homomorphism if we choose ab $\left(x_{i}\right)$ as the $C$-generators of $G / G^{\prime}$, where the set $\left\{x_{i}\right\}$ is the set of $C$-generators of the group $G$. We say that a $C$-group $G$ consists of $n$ irreducible components if $G / G^{\prime} \simeq \mathbb{Z}^{n}$. The notion of the number of irreducible components of a Hurwitz $C$-group is explained by the following simple lemma.

Lemma 5.7. A (topological) Hurwitz curve $\bar{H}$ consists of $n$ irreducible components iff its fundamental group $\pi_{1}=\pi_{1}\left(\mathbb{C}^{2} \backslash H\right)$ consists of $n$ irreducible components.

Trivial computations show that the Alexander polynomial $\Delta(t)$ of an abelian $C$-group $\mathbb{Z}^{n}$ is equal to $(-1)^{n-1}(t-1)^{n-1}$. Therefore, Lemma 5.5 implies the following

Lemma 5.8. The Alexander polynomial $\Delta(t)$ of a Hurwitz $C$-group $G$ consisting of $n$ irreducible components is divisible by $(t-1)^{n-1}$.

Theorem 5.9. Let a Hurwitz $C$-group $G$ consist of $n$ irreducible components and $\Delta(t)$ be its Alexander polynomial. Then

$$
\Delta(t)=(t-1)^{n-1} P(t),
$$

where the polynomial $P(t) \in \mathbb{Z}[t]$ satisfies $P(1) \neq 0$.

Proof. Let $m$ be the degree of the Hurwitz $C$-group $G$. To obtain a $C$-presentation of $G$, it is sufficient to add several $C$-relations to presentation (23).

Since $G$ consists of $n$ irreducible components, the set $\{1, \ldots, m\}$ splits into a disjoint union of $n$ subsets $J_{1}, \ldots, J_{n}$ such that $j_{1}, j_{2} \in J_{k}$ iff $x_{j_{1}}$ and $x_{j_{2}}$ are conjugated in $G$. Let $J_{k}=\left\{j_{1, k}<\cdots<j_{m_{k}, k}\right\}$. Without loss of generality, one can assume that among the added $C$ relations there are relations

$$
x_{j_{i, k}} w_{j_{i, k}, j_{i+1, k}, 1}=w_{j_{i, k}, j_{i+1, k}, 1} x_{j_{i+1, k}}
$$

for $i=1, \ldots, m_{k}-1, k=1, \ldots, n$, and for some words $w_{j_{i, k}, j_{i+1, k}, 1}$. Let $\nu\left(w_{j_{i, k}, j_{i+1, k}, 1}\right)=x^{t_{j_{i, k}}}$, where $x$ is the $C$-generator of $\mathbb{F}_{1}$.

Consider diagram (*) in which $G_{1}=\widetilde{G}_{m}$ and $G_{2}=G$. In the notations of the proof of Theorem [5.6. the elements $\bar{a}_{0, m}$ and $\sum_{k=1}^{m} \bar{a}_{k, j}$, $j=2, \ldots, m-1$, generate the eigenspace $\left(N_{1} / N_{1}^{\prime}\right)_{1}$ of $N_{1} / N_{1}^{\prime}$ corresponding to the eigenvalue 1 . Its image $g_{*}\left(\left(N_{1} / N_{1}^{\prime}\right)_{1}\right) \otimes \mathbb{C}$ is the eigenspace of $N_{2} / N_{2}^{\prime} \otimes \mathbb{C}$ corresponding to the eigenvalue 1 . 
Applying the Reidemeister - Schreier method, the element $a_{0, m}$, defined in (25), and the elements $a_{k, j}, k=1, \ldots, m, j=2, \ldots, m-1$, defined in (24) (more precisely, their images under $g_{*}$ ), also generate the group $N_{2}$ and each relation (28) (after the substitution $x_{1}=$ $\left.y\left(x_{2} \ldots x_{m}\right)^{-1}\right)$ gives rise to the relations

$$
a_{r, j_{i, k}} \bar{w}_{r+1, j_{i, k}, j_{i+1, k}}=\bar{w}_{r, j_{i, k}, j_{i+1, k}} a_{r+t_{j_{i, k}}, j_{i+1, k}}
$$

if $1<j_{i, k}<j_{i+1, k}<m$,

$$
a_{0, m}\left(\prod_{s=2}^{m-1} a_{r+m-s, m+1-s}^{-1}\right) \bar{w}_{r+1,1, j_{i+1, k}}=\bar{w}_{r, 1, j_{i+1, k}} a_{r+t_{1, j_{i+1, k}}}
$$

if $1=j_{i, k}<j_{i+1, k}<m$,

$$
a_{r, j_{i, k}} \bar{w}_{r+1, j_{i, k}, m}=\bar{w}_{r, j_{i, k}, m}
$$

if $1<j_{i, k}<j_{i+1, k}=m$, and

$$
a_{0, m}\left(\prod_{s=2}^{m-1} a_{r+m-s, m+1-s}^{-1}\right) \bar{w}_{r+1,1, m}=\bar{w}_{r, 1, m}
$$

if $1=j_{i, k}<j_{i+1, k}=m$, where $r \in \mathbb{Z}$ and each word $\bar{w}_{r, j_{i, k}, j_{i+1, k}}=$ $x_{m}^{r} w_{j_{i, k}, j_{i+1, k}, 1} x_{m}^{-\left(r+t_{j_{i, k}}\right)}$ is written in the generators $a_{l, s}$.

As in [13, one can show that the words $\bar{w}_{r, j_{i, k}, j_{i+1, k}}$ and $\bar{w}_{r+m, j_{i, k}, j_{i+1, k}}$ are conjugated in $G_{2}$ by $a_{0, m}$. Therefore, taking the sum over $r$, relations (29) - (32) give rise in $N_{2} / N_{2}^{\prime}$ to the following relations:

$$
\sum_{r=1}^{m} \bar{a}_{r, j_{i, k}}=\sum_{r=1}^{m} \bar{a}_{r, j_{i+1, k}}
$$

if $1<j_{i, k}<j_{i+1, k}<m$,

$$
m \bar{a}_{0, m}-\sum_{s=2}^{m-1} \sum_{r=1}^{m} \bar{a}_{r, s}=\sum_{r=1}^{m} \bar{a}_{r, j_{i+1, k}}
$$

if $1=j_{i, k}<j_{i+1, k}<m$,

$$
\sum_{r=1}^{m} \bar{a}_{r, j_{i, k}}=0
$$

if $1<j_{i, k}<j_{i+1, k}=m$, and

$$
m \bar{a}_{0, m}-\sum_{s=2}^{m-1} \sum_{r=1}^{m} \bar{a}_{r, s}=0
$$

if $1=j_{i, k}<j_{i+1, k}=m$.

It is easy to see that for any decomposition $\{1, \ldots, m\}=\bigsqcup_{k=1}^{n} J_{k}$, equations (33) - (36) are linear independent over $\mathbb{Z}$, their number being 
$m-n$, and the elements $\bar{a}_{0, m}$ and $\sum_{r=1}^{m} \bar{a}_{r, s}, s=2, \ldots, m-1$ generate the eigenspace $\left(N_{1} / N_{1}^{\prime}\right)_{1}$. Therefore the rank of the kernel of the restriction of $g_{*}$ to $\left(N_{1} / N_{1}^{\prime}\right)_{1}$ is not less then $m-n$. Thus

$$
\operatorname{dim}\left(N_{2} / N_{2}^{\prime} \otimes \mathbb{C}\right)_{1} \leqslant \operatorname{dim}\left(N_{1} / N_{1}^{\prime} \otimes \mathbb{C}\right)_{1}-(m-n)=n-1 .
$$

On the other hand, by Lemma [5.8, the Alexander polynomial $\Delta(t)$ of a $C$-group $G$ consisting of $n$ irreducible components is divisible by $(t-1)^{n-1}$.

Corollary 5.10. Let a Hurwitz $C$-group $G$ consist of $n$ irreducible components and $\Delta(t)$ be its Alexander polynomial. Then

$$
\Delta(0)=(-1)^{\operatorname{deg} \Delta(t)-(n-1)} .
$$

Proof. The Alexander polynomial

$$
\Delta(t)=\operatorname{det}\left(h_{\mathbb{C}}-t \mathrm{Id}\right)=(-t)^{\operatorname{deg} \Delta(t)}+\sum_{i=0}^{\operatorname{deg} \Delta(t)-1} c_{i} t^{i}
$$

satisfies, and by Theorem [5.9], $\Delta(t)=(t-1)^{n-1} P(t)$ where the polynomial $P(t) \in \mathbb{Z}[t]$ is such that $P(1) \neq 0$. Therefore the polynomial $P(t)=(-1)^{\operatorname{deg} \Delta(t)} \prod_{i} \Phi_{n_{i}}(t)$ is a product (up to sign) of some cyclotomic polynomials $\Phi_{n_{i}}(t)$ with $n_{i}>1$. By Lemma 5.3, $\Phi_{n_{i}}(0)=1$ for all $n_{i}>1$. Therefore $\Delta(0)=(-1)^{n-1}(-1)^{\Delta(t)}=(-1)^{\operatorname{deg} \Delta(t)-(n-1)}$.

Lemma 5.11. Let $j:\{1, \ldots, n\} \rightarrow\{1, \ldots, m\}$ be an injective function. Assume that a $C$-group $G$ is generated by $C$-generators $x_{1}, \ldots, x_{n}$ and $w=\bar{x}_{1} \ldots \bar{x}_{m}$ is a quasipositive word in $x_{1}, \ldots, x_{n}$ (that is, each $\bar{x}_{k}$ is conjugated to some $\left.x_{i_{k}} \in\left\{x_{1}, \ldots, x_{n}\right\}\right)$ such that $\bar{x}_{j(i)}=x_{i}$ for $i=$ $1, \ldots, n$. If $w$ belongs to the center of $G$, then $G$ is a Hurwitz $C$-group of degree $m$.

Proof. Let $G=<x_{1}, \ldots, x_{n} \mid R>$ be a $C$-presentation of the group $G$. Put $J=\{1 \leqslant j \leqslant m \mid j=j(i), i=1, \ldots, n\}$. By assumption, we have $\bar{x}_{j(i)}=x_{i}$ and

$$
\bar{x}_{j}=w_{j}^{-1} x_{i_{j}} w_{j}
$$

for $j \notin J$, where $w_{j}$ is a word in $x_{1}, \ldots, x_{n}$. Note that relations (37) are $C$-relations. Therefore if we add the generators $\bar{x}_{j}, j \notin J$, to the set of generators $\left\{x_{1}, \ldots, x_{n}\right\}$ and add relations (37) to $R$, then we obtain a $C$-presentation of the same group $G$. To complete the proof, it suffices to renumber the obtained set of generators.

Proposition 5.12. Let $G_{i}, i=1,2$, be a Hurwitz $C$-group of degree $m_{i}$ and $\Delta_{i}(t)$ its Alexander polynomial. Then there exists a Hurwitz $C$ group of degree $2 m_{1} m_{2}$ with Alexander polynomial $\Delta(t)=\Delta_{1}(t) \Delta_{2}(t)$. 
Proof. Let $G_{i}=<x_{1, i}, \ldots, x_{m_{i}, i} \mid R_{i}>$ be a Hurwitz $C$-presentation of the group $G_{i}$ of degree $m_{i}$.

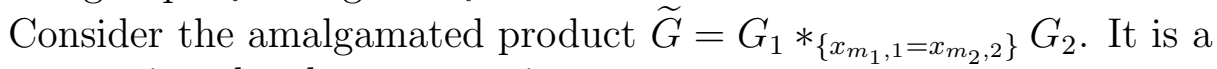
$C$-group given by the presentation

$$
\widetilde{G}=<x_{1, i}, \ldots, x_{m_{i}, i}, i=1,2 \mid R_{1} \cup R_{2}, x_{m_{1}, 1}=x_{m_{2}, 2}>.
$$

Put $y_{i}=x_{1, i} \ldots x_{m_{i}, i}, i=1,2$, and denote by $N_{i}$ (resp. $\left.\tilde{N}\right)$ the kernel of $\nu: G_{i} \rightarrow \mathbb{F}_{1}$ (resp. of $\nu: \widetilde{G} \rightarrow \mathbb{F}_{1}$ ).

As in the proof of Theorem 5.6, applying the Reidemeister - Schreier method, one can show that the group $N_{i}$ (resp. $\left.\widetilde{N}\right)$ is generated by the elements $a_{k, j, i}=x_{m_{i}, i}^{k} x_{j, i} x_{m_{i}, i}^{-(k+1)}$, where $j=2, \ldots, m_{i}-1, k \in \mathbb{Z}$, and by the elements $a_{k, m_{i}, i}=x_{m_{i}, i}^{k} y_{i} x_{m_{i}, i}^{-\left(k+m_{i}\right)}, k \in \mathbb{Z}$ (resp. by the union of these elements, since $x_{m_{1}, 1}=x_{m_{2}, 2}$ in $\left.\widetilde{G}\right)$. The set of defining relations of $N_{i}$ (resp. of $\tilde{N}$ ) is obtained from the set $\bar{R}_{i}=\left\{x_{m_{i}, i}^{n} r_{l, i} x_{m_{i}, i}^{-n} \mid r_{l, i} \in\right.$ $\left.R_{i} n \in \mathbb{Z}\right\}$ (resp. from $\bar{R}=\bar{R}_{1} \cup \bar{R}_{2}$ ) after rewriting the words in the alphabet $\left\{a_{k, j, i}\right\}$ (resp. in the union of these alphabets). Therefore $\widetilde{N}=N_{1} * N_{2}$ is the free product of the groups $N_{1}$ and $N_{2}$.

Note that it follows from the proof of Theorem 5.6 that the elements $a_{k, j, i}$ and $a_{k+l m_{i}, j, i}$ are conjugated in $N_{i}$ for all $l \in \mathbb{Z}$.

Let $\widetilde{h}_{i}$ be the automorphism of $N_{i}$ given by conjugation by $x_{m_{i}, i}$. Then the automorphism $\widetilde{h}$ of $\widetilde{N}$ given by conjugation by $x_{m_{1}, 1}=x_{m_{2}, 2}$, which is equal to $\widetilde{h}_{1} * \widetilde{h}_{2}$. Therefore the Alexander polynomial $\widetilde{\Delta}(t)$ of the group $\widetilde{G}$ is equal to $\widetilde{\Delta}(t)=\Delta_{1}(t) \Delta_{2}(t)$.

Consider a group

$$
\begin{gathered}
G=<x_{1, i} \ldots, x_{m_{i}, i}, i=1,2 \mid R_{1} \cup R_{2}, x_{m_{1}, 1}=x_{m_{2}, 2}, \\
{\left[x_{j, i}, y_{\bar{i}}^{m_{i}}\right]=1, j=1, \ldots, m_{i}-1, i=1,2>,}
\end{gathered}
$$

where $\bar{i}=\{1,2\} \backslash\{i\}$ (recall that $x_{1, \bar{i}}, \ldots, x_{m_{\bar{i}}, \bar{i}}$ commute with $y_{\bar{i}}$ ). Let $N$ be the kernel of $\nu: G \rightarrow \mathbb{F}_{1}$.

To obtain a presentation of the group $N$ from the presentation of the group $\widetilde{N}$ described above, one should add the relations induced by the relations

$$
\left[x_{j, i}, y_{\bar{i}}^{m_{i}}\right]=1, j=1, \ldots, m_{i}-1, i=1,2 .
$$

It is easy to see that these additional relations are

$$
a_{k, j, i} a_{0, m_{\bar{i}}}^{m_{i}}=a_{0, m_{\bar{i}}}^{m_{i}} a_{k+m_{1} m_{2}, j, i}
$$

since $a_{k, m_{\bar{i}}}=a_{k+1, m_{\bar{i}}}$ in $N_{\bar{i}}$ for all $k$, that is, the additional relations (38) say that $a_{k, j, i}$ and $a_{k+m_{1} m_{2}, j, i}$ are conjugated. But these elements were conjugated in $\tilde{N}$. Therefore $\tilde{N} / \widetilde{N}^{\prime} \simeq N / N^{\prime}$ and the groups $\widetilde{G}$ and $G$ 
have the same Alexander polynomials. To complete the proof of Proposition 5.12, let us notice that $y_{1}^{m_{2}} y_{2}^{m_{1}}=\left(x_{1,1} \ldots x_{m_{1}, 1}\right)^{m_{2}}\left(x_{1,2} \ldots x_{m_{2}, 2}\right)^{m_{1}}$ belongs to the center of the group $G$. Therefore, by Lemma 5.11, $G$ is a Hurwitz $C$-group of degree $2 m_{1} m_{2}$.

For two Hurwitz $C$-groups $G_{1}$ and $G_{2}$ given by Hurwitz $C$-presentations $G_{i}=<x_{1, i}, \ldots, x_{m_{i}, i} \mid R_{i}>, i=1,2$, the Hurwitz $C$-group $G$, constructed in the proof of Proposition 5.12 , is called a Hurwitz product of $G_{1}$ and $G_{2}$. A Hurwitz product of $G_{1}$ and $G_{2}$ will be denoted by $G_{1} \diamond G_{2}$. Of course, a Hurwitz product of $G_{1}$ and $G_{2}$ depends on Hurwitz $C$-presentations of $G_{1}$ and $G_{2}$, but by Proposition 5.12, the Alexander polynomial of $G_{1} \diamond G_{2}$ does not depend on the Hurwitz $C$-presentations of the factors.

Lemma 5.13. The fundamental group $G_{n, m}=\pi_{1}\left(\mathbb{C}^{2} \backslash C_{n, m}\right)$ of the complement of the affine plane algebraic curve $C_{m, n}$, given by the equation $w^{n}-z^{m}=0$, where $n$ and $m$ are any positive integers, is a Hurwitz C-group.

Remark. Note that this lemma does not follow from the statement, mentioned above, on the fundamental group of the complement of an affine Hurwitz curve, since it is assumed there that the line at infinity is in general position with respect to the Hurwitz curve. Here, the line at infinity is in special position. If we consider the local fundamental group $G=\pi_{1}\left(B_{\varepsilon} \backslash C\right)$, where $C$ is an irreducible singularity in a small ball $B_{\varepsilon}$, then $G$ has always a natural structure of an irreducible $C$ group. It has a non-trivial center iff the singularity $C$ is of type $x^{p}=y^{q}$ with $p$ and $q$ coprime (see [3]).

Proof. Indeed, a braid monodromy of the singularity $w^{n}=z^{m}$ with respect to the projection $(z, w) \rightarrow z$ is equal to

$$
b_{n, m}=\left(\sigma_{1} \ldots \sigma_{n-1}\right)^{m} \in \mathrm{Br}_{n},
$$

where $\sigma_{1}, \ldots, \sigma_{n-1}$ are standard generators of the braid group $\mathrm{Br}_{n}$, that is, the generators satisfy the following relations $\sigma_{i} \sigma_{i+1} \sigma_{i}=\sigma_{i+1} \sigma_{i} \sigma_{i+1}$ for $i=1, \ldots, n-2$ and $\left[\sigma_{i}, \sigma_{j}\right]=1$ for $|i-j| \geqslant 2$. The group $\operatorname{Br}_{n}$ acts on the free group $\mathbb{F}_{n}$ generated by $x_{1}, \ldots, x_{n}$. This action is given by $\sigma_{j}\left(x_{i}\right)=x_{i}$ if $j \neq i, i+1, \sigma_{j}\left(x_{j+1}\right)=x_{j}$, and $\sigma_{j}\left(x_{j}\right)=x_{j} x_{j+1} x_{j}^{-1}$. Denote by $B_{n, m}$ the cyclic subgroup of $\mathrm{Br}_{n}$ generated by $b_{n, m}$. Then (see [12]) the group

$$
G_{n, m}=<x_{1}, \ldots, x_{n} \mid x_{i}=b\left(x_{i}\right), i=1, \ldots, n, b \in B_{n, m}>
$$

is a $C$-group, and by Lemma [5.11, it is a Hurwitz $C$-group, since $b_{n, m}^{n}=\left(\Delta_{n}^{2}\right)^{m} \in B_{n, m}$, where $\Delta_{n}$ is the Garside element of the braid 
group $\mathrm{Br}_{n}$, and therefore the element $\left(x_{1} \ldots x_{n}\right)^{m}$ belongs to the center of $G_{n, m}\left(\right.$ since $\Delta_{n}^{2}\left(x_{i}\right)=\left(x_{1} \ldots x_{n}\right) x_{i}\left(x_{1} \ldots x_{n}\right)^{-1}$ and $\Delta_{n}^{2}\left(x_{1} \ldots x_{n}\right)=$ $\left.x_{1} \ldots x_{n}\right)$.

Proposition 5.14. ([14]) If $m$ and $n$ are coprime, then the group $G_{n, m}$ has the Alexander polynomial

$$
\Delta_{n, m}(t)=\frac{(t-1)\left(t^{n m}-1\right)}{\left(t^{n}-1\right)\left(t^{m}-1\right)}
$$

Proposition 5.15. The Alexander polynomial of the group $G_{2,2 m}$ is equal to

$$
\Delta(t)=(1-t) \sum_{i=0}^{m-1} t^{2 i} .
$$

Proof. It follows from the proof of Lemma 5.13 that

$$
G_{2,2 m}=<x_{1}, x_{2} \mid\left[x_{1},\left(x_{1} x_{2}\right)^{m}\right]=\left[x_{2},\left(x_{1} x_{2}\right)^{m}\right]=1>.
$$

Let us show that the relations

$$
\left[x_{2},\left(x_{1} x_{2}\right)^{m}\right]=1, \quad i=1,2,
$$

are equivalent to the single relation

$$
\left(x_{1} x_{2}\right)^{m}=\left(x_{2} x_{1}\right)^{m} \text {. }
$$

Indeed, relations (39) imply

$$
\left(x_{1} x_{2}\right)^{m}=x_{1}^{-1}\left(x_{1} x_{2}\right)^{m} x_{1}=\left(x_{2} x_{1}\right)^{m},
$$

the relation $\left(x_{1} x_{2}\right)^{m}=\left(x_{2} x_{1}\right)^{m}$ implies

$$
x_{2}\left(x_{1} x_{2}\right)^{m}=\left(x_{2} x_{1}\right)^{m} x_{2}=\left(x_{1} x_{2}\right)^{m} x_{2}
$$

and

$$
x_{1}\left(x_{1} x_{2}\right)^{m}=x_{1}\left(x_{2} x_{1}\right)^{m}=\left(x_{1} x_{2}\right)^{m} x_{1} .
$$

Therefore

$$
G_{2,2 m}=<x_{1}, x_{2} \mid\left(x_{1} x_{2}\right)^{m}\left(x_{2} x_{1}\right)^{-m}=1>.
$$

Denote by $r=\left(x_{1} x_{2}\right)^{m}\left(x_{2} x_{1}\right)^{-m}$. Applying the free differential calculus of Fox (4]), it is easy to see that

$$
\nu_{*}\left(\frac{\partial r}{\partial x_{1}}\right)=1+t^{2}+\cdots+t^{2(m-1)}-t^{2 m-1}-t^{2 m-3}-\cdots-t^{1}
$$

and

$$
\nu_{*}\left(\frac{\partial r}{\partial x_{2}}\right)=t+\cdots+t^{2 m-1}-t^{2(m-1)}-t^{2(m-2)}-\cdots-t^{2}-1 .
$$


Therefore

$$
\Delta(t)=(1-t) \sum_{i=0}^{m-1} t^{2 i}
$$

Consider the group

$$
\begin{aligned}
G(2)=<x_{1}, \ldots, x_{4} \mid & x_{2}^{2} x_{1} x_{2}^{-2}=x_{4}, x_{3}=x_{2}, x_{4}^{2} x_{2} x_{4}^{-2}=x_{2} \\
& {\left[x_{i}, x_{1} \ldots x_{4}\right]=1 \text { for } i=1, \ldots, 4>. }
\end{aligned}
$$

Proposition 5.16. The Alexander polynomial of the group $G(2)$ is equal to $\Delta(t)=t^{2}-1$.

Proof. Denote by $N(2)$ the kernel of $\nu: G(2) \rightarrow \mathbb{F}_{1}$ and put $m=4$ and $y=x_{1} \ldots x_{4}$. In the notations of the proof of Theorem [5.6, it follows from the relations $\left[x_{i}, y\right]=1$ for $i=1, \ldots, 4$ that the group $N(2)$ is generated by the elements $a_{k, j}=x_{4}^{k} x_{j} x_{4}^{-(k+1)}, k=1, \ldots, 4, j=2,3$, and the element $a_{0,4}=y x_{4}^{-4}$. In our case relations (26) and (27) have the form

$$
a_{k, 4}=a_{0,4} \quad \text { and } \quad a_{k+4, j}=a_{0,4}^{-1} a_{k, j} a_{0,4}
$$

for all $k$.

The relation $x_{3}=x_{2}$ gives rise to the relations

$$
a_{k, 3}=a_{k, 2}
$$

for all $k$.

The relation $x_{4}^{2} x_{2}=x_{2} x_{4}^{2}$ gives rise to the relations

$$
a_{k+2,2}=a_{k, 2}
$$

for all $k$.

The relation $x_{2}^{2} x_{1}=x_{4} x_{2}^{2}$, written as $x_{2}^{2} y=x_{4} x_{2}^{4} x_{4}$ ( since $\left.x_{2}=x_{3}\right)$, gives rise to the relations

$$
a_{k, 2} a_{k+1,2} a_{k+2,4}=a_{k+1,2} a_{k+2,2} a_{k+3,2} a_{k+4,2}
$$

for all $k$.

It follows from (41) - (44) that $N(2)$ is generated by $a_{1,2}, a_{2,2}$ and $a_{0,4}$, being subject to the relations

$$
a_{0,4}=\left(a_{1,2} a_{2,2}\right)^{-1}\left(a_{2,2} a_{1,2}\right)^{2}=\left(a_{2,2} a_{1,2}\right)^{-1}\left(a_{1,2} a_{2,2}\right)^{2}
$$

and

$$
\left[a_{1,2}, a_{0,4}\right]=\left[a_{2,2}, a_{0,4}\right]=1 .
$$

Therefore the group $N(2) / N(2)^{\prime}$ is a free abelian group generated by the images $\bar{a}_{1,2}$ and $\bar{a}_{2,2}$ of the elements $a_{1,2}$ and $a_{2,2}$. 
As in the proof of Theorem [5.6, the action $\widetilde{h}$ on $N(2)$ is given by $\widetilde{h}\left(a_{1,2}\right)=a_{2,2}, \widetilde{h}\left(a_{2,2}\right)=a_{3,2}=a_{1,2}$. The induced action $h$ on $N(2) / N(2)^{\prime}$ is given by $h\left(\bar{a}_{1,2}\right)=\bar{a}_{2,2}$ and $h\left(\bar{a}_{2,2}\right)=\bar{a}_{1,2}$ the characteristic polynomial of which is equal to $(t-1)(t+1)$.

Corollary 5.17. For any $k \in \mathbb{N}$ there is a Hurwitz $C$-group $G$ consisting of two irreducible components whose Alexander polynomial $\Delta(t)=$ $(t-1) P(t)$ is such that $|P(1)|=k$.

Proof. If $k>2$ then, by Proposition 5.15, we have $P(1)=-k$, where $P(t)=-\sum_{i=0}^{k-1} t^{2 i}$ is the factor of the Alexander polynomial $\Delta(t)=$ $(1-t) \sum_{i=0}^{k-1} t^{2 i}$ of the group $G_{2,2 k}$. If $k=2$, then, by Proposition 5.16. the group $G(2)$ has the desired property, since its Alexander polynomial is $\Delta(t)=(t-1)(t+1)$. In the case $k=1$, one can take the abelian Hurwitz $C$-group $G=\mathbb{Z}^{2}$.

Proposition 5.18. For any $k \in \mathbb{N}$ there exists

(i) an irreducible Hurwitz $C$-group whose Alexander polynomial $\Delta(t)$ has $\operatorname{deg} \Delta(t)=2 k$;

(ii) a Hurwitz $C$-group consisting of two irreducible components and whose Alexander polynomial $\Delta(t)=(t-1) P(t)$ satisfies $\operatorname{deg} P(t)=k$.

Proof. By Propositions 5.12 and 5.14, the Alexander polynomial $\Delta(t)$ of a Hurwitz product $G_{2,3}^{\diamond k}$ is equal to $\left(t^{2}-t+1\right)^{k}$.

To prove $(i i)$, it suffices to take the groups $G(2) \diamond G_{2,3}^{\diamond n}$ if $k=2 n+1$ is odd and $\mathbb{Z}^{2} \diamond G_{2,3}^{\diamond n}$ if $k=2 n$ is even.

Question 5.19. Let $P(t) \in \mathbb{Z}[t]$ be a polynomial whose roots are roots of unity, let $t=1$ be a root of $P(t)$ of multiplicity $k$, and $P(0)=$ $(-1)^{\operatorname{deg} P(t)-k}$. Assume also that $P(1)=1$ if $k=0$. Does there exist a Hurwitz $C$-group $G$ with Alexander polynomial $\Delta(t)=P(t)$ ?

\section{The first Betti number of CYCliC COVERINGS OF THE} PLANE

Consider the infinite cyclic covering $f=f_{\infty}: X_{\infty} \rightarrow X^{\prime}=\mathbb{C}^{2} \backslash H$ corresponding to the epimorphism $\nu: \pi_{1}\left(\mathbb{C}^{2} \backslash H\right) \rightarrow \mathbb{F}_{1}$. Let $h \in$ $\operatorname{Deck}\left(X_{\infty} / X^{\prime}\right) \simeq \mathbb{F}_{1}$ be a covering transformation corresponding to the $C$-generator $x \in \mathbb{F}_{1}$. We say that $h$ is the monodromy of a Hurwitz curve $H$. The space $X^{\prime}$ will be considered as the quotient space $X^{\prime}=$ $X_{\infty} / \mathbb{F}_{1}$. In such a situation Milnor [17] considered an exact sequence of chain complexes

$$
0 \rightarrow C .\left(X_{\infty}\right) \stackrel{h-i d}{\longrightarrow} C .\left(X_{\infty}\right) \stackrel{\varphi_{*}}{\longrightarrow} C .\left(X^{\prime}\right) \rightarrow 0
$$


which gives an exact homology sequence

$$
\ldots \rightarrow H_{1}\left(X_{\infty}\right) \stackrel{h-i d}{\longrightarrow} H_{1}\left(X_{\infty}\right) \stackrel{f_{*}}{\longrightarrow} H_{1}\left(X^{\prime}\right) \rightarrow H_{0}\left(X_{\infty}\right) \rightarrow 0
$$

(We often write $h$ instead of $h_{*}$, if it does not lead to a misunderstanding).

If $G_{n} \subset \mathbb{F}_{1}$ is an infinite cyclic group generated by $h^{n}$, then $X_{n}^{\prime}=$ $X_{\infty} / G_{n}$ and $X^{\prime}=X_{n}^{\prime} / \mu_{n}$, where $\mu_{n}=\mathbb{F}_{1} / G_{n}$ is the cyclic group of order $n$. Denote by $h_{n}$ an automorphism of $X_{n}^{\prime}$ induced by the monodromy $h$. Then $h_{n}$ is a generator of the covering transformation group $\operatorname{Deck}\left(X_{n}^{\prime} / X^{\prime}\right)=\mu_{n}$ acting on $X_{n}^{\prime}$. We apply the sequence

$$
\ldots \rightarrow H_{1}\left(X_{\infty}\right) \stackrel{h^{n}-i d}{\longrightarrow} H_{1}\left(X_{\infty}\right) \stackrel{g_{n, *}}{\longrightarrow} H_{1}\left(X_{n}^{\prime}\right) \rightarrow H_{0}\left(X_{\infty}\right) \rightarrow 0
$$

constructed in the same way as (45) to the infinite cyclic covering $g_{n}=g_{\infty, n}: X_{\infty} \rightarrow X_{n}^{\prime}$, to analyse the group $H_{1}\left(X_{n}^{\prime}\right)$.

Denote by $H_{1}\left(X_{\infty}, \mathbb{C}\right)_{n}$ the subspace of $H_{1}\left(X_{\infty}, \mathbb{C}\right)$ corresponding to the eigenvalues $\lambda$ of $h_{*}$ which are $n$-th roots of unity and denote by $H_{1}\left(X_{\infty}, \mathbb{C}\right)_{n, \neq 1}$ the subspace corresponding to the eigenvalues $\lambda \neq 1$ of $h$ which are $n$-th roots of unity. Obviously, $\operatorname{dim} H_{1}\left(X_{\infty}, \mathbb{C}\right)_{n}=r_{n}$ and $\operatorname{dim} H_{1}\left(X_{\infty}, \mathbb{C}\right)_{n, \neq 1}=r_{n, \neq 1}$, where $r_{n}$ (resp. $r_{n, \neq 1}$ ) is the number of roots of the Alexander polynomial $\Delta(t)$ of the Hurwitz curve $\bar{H}$ which are $n-t h$ roots of unity (resp. not equal to 1 ). Note that by Lemma 5.7 and Theorem [5.9.

$$
r_{n}-r_{n, \neq 1}=r_{1}=\#\{\text { irreducible components of } \bar{H}\}-1 \text {. }
$$

Proposition 6.1. We have

(i) $\operatorname{dim} H_{1}\left(X_{n}^{\prime}, \mathbb{C}\right)=r_{n}+1$,

(ii) $\operatorname{dim} H_{1}\left(X_{n}^{\prime}, \mathbb{C}\right)_{1}=r_{1}+1=\#\{$ irreducible components of $\bar{H}\}$.

Proof. This follows from the exact sequence (46).

Let $\bar{H}$ be a Hurwitz curve consisting of $k$ irreducible components $\bar{H}_{1}, \ldots, \bar{H}_{k}$. Choose a line $L \subset \mathbb{C}^{2}$ belonging to the pencil of lines (with respect to which $\bar{H}$ is defined) and transversely intersecting the curve $H$. Denote by $\gamma_{i}$ a circle of small radius in $L$ with center at one of the intersection points $H_{i} \cap L$. It is easy to see that the cycles $\gamma_{1}, \ldots, \gamma_{k}$, corresponding to the chosen loops, form a basis in $H_{1}\left(\mathbb{C}^{2} \backslash H, \mathbb{Z}\right)$ and are independent of the choice of the line $L$. Let $\bar{\gamma}_{i}, i=1, \ldots, k$, be a cycle in $H_{1}\left(X_{n}^{\prime}, \mathbb{Z}\right)$ corresponding to a simple path $f_{n}^{-1}\left(\gamma_{i}\right)$.

Lemma 6.2. The cycles $\bar{\gamma}_{i}, i=1, \ldots, k$, are linear independent in $H_{1}\left(X_{n}^{\prime}, \mathbb{Z}\right)$ and form a basis in $H_{1}\left(X_{n}^{\prime}\right)_{1}$. 
Proof. Obviously, all $\bar{\gamma}_{i}$ are invariant under the action $h_{n}$. Now the proof follows from Proposition 6.1 $(i i)$ and from the remark that under the homomorphism $\left(f_{n}\right)_{*}: H_{1}\left(X_{n}^{\prime}, \mathbb{Z}\right) \rightarrow H_{1}\left(\mathbb{C}^{2} \backslash H, \mathbb{Z}\right)$ we have $\left(f_{n}\right)_{*}\left(\bar{\gamma}_{i}\right)=n \gamma_{i}$.

In the notations of the proof of Theorem 4.1, the covering $f_{n}$ can be extended to a map $\widetilde{f}_{n}: \widetilde{X}_{n} \rightarrow \mathbb{C P}^{2}$ branched along $\bar{H}$ and, maybe, along $\bar{L}_{\infty}$. Here $\widetilde{X}_{n}$ is a closed four dimensional variety locally isomorphic over a singular point of $\bar{H}$ to a complex analytic singularity given by an equation $w_{1}^{n}=\widetilde{F}_{1}\left(u_{1}, v_{1}\right)$, where $\widetilde{F}_{1}\left(u_{1}, v_{1}\right)=\prod\left(v_{1}-v_{1, j}\left(u_{1}\right)\right)$ and the product is taken over those branches of $\bar{H}$ for which the closure contains the singular point of $\bar{H}$. In addition, $\widetilde{X}_{n}$ is locally isomorphic over a neighbourhood of an intersection point of $\bar{H}$ and $L_{\infty}$ to the singularity given by $w_{2}^{n}=\left(v_{2}-e^{\frac{2 \pi i}{m}}\right) u^{d}$, where $d$ is the smallest non-negative integer for which $m+d$ is divisible by $n$. The variety $\widetilde{X}_{n}$, if $\widetilde{f}_{n}^{-1}\left(L_{\infty}\right) \subset \operatorname{Sing} \widetilde{X}_{n}$, can be normalized (as in the algebraic case) and we obtain a covering $\widetilde{f}_{n, \text { norm }}: \widetilde{X}_{n, \text { norm }} \rightarrow \mathbb{C P}^{2}$ in which $\tilde{X}_{n, \text { norm }}$ is a singular analytic variety at its finitely many singular points. One can resolve them and obtain a smooth manifold $\bar{X}_{n}$. Let $\sigma: \bar{X}_{n} \rightarrow \widetilde{X}_{n \text {,norm }}$ be a resolution of the singularities, $E=\sigma^{-1}\left(\operatorname{Sing} \widetilde{X}_{n, \text { norm }}\right)$, and $\bar{f}_{n}=\widetilde{f}_{n \text {, norm }} \circ \sigma$. Denote by $R_{i}=\widetilde{f}_{n, \text { norm }}^{-1}\left(\bar{H}_{i}\right), i=1, \ldots, k$, and $R_{\infty}=\widetilde{f}_{n, \text { norm }}^{-1}\left(L_{\infty}\right)$. Note that the restriction of $\widetilde{f}_{n \text {,norm }}$ to each $R_{i}, i=1, \ldots, k$, is one-to-one and the restriction of $\widetilde{f}_{n \text {, norm }}$ to $R_{\infty}$ is a $n_{0}$-sheeted cyclic covering, where $n_{0}=\operatorname{GCD}(n, d)$ and the ramification index of $\tilde{f}_{n \text {, norm }}$ along $R_{\infty}$ is equal to $n_{\infty}=\frac{n}{n_{0}}$. As in the algebraic case, it is easy to show that $R_{\infty}$ is irreducible. Denote by $\bar{R}_{i}=\sigma^{-1}\left(R_{i}\right), i=1, \ldots, k, \infty$, the proper transform of $R_{i}$.

We have the embeddings $i_{1}: X_{n}^{\prime} \hookrightarrow X_{n}=\bar{X}_{n} \backslash E$ and $i_{2}: X_{n} \hookrightarrow \bar{X}_{n}$.

Lemma 6.3. The induced homomorphism $i_{1 *}: H_{1}\left(X_{n}^{\prime}\right) \rightarrow H_{1}\left(X_{n}\right)$ is an epimorphism with $\operatorname{ker} i_{1 *}=H_{1}\left(X_{n}^{\prime}\right)_{1}$.

Proof. We have

$$
X_{n}^{\prime}=X_{n} \backslash\left(\cup_{i=1}^{k} \bar{R}_{i}\right) \cup \bar{R}_{\infty}
$$

and each $\bar{R}_{i}, i=1, \ldots, k, \infty$, is a codimension two submanifold of $X_{n}$. Therefore each 1-dimensional cycle $\gamma \subset X_{n}$ can be moved outside of $\left(\cup_{i=1}^{k} \bar{R}_{i}\right) \cup \bar{R}_{\infty}$. Thus, $i_{1, *}$ is an epimorphism.

Let a complex line $L \subset \mathbb{C P}^{2}$ meet $L_{\infty}$ transversely at $q \in L_{\infty} \backslash \bar{H}$ and $\gamma_{\infty}$ be a simple small loop around $L_{\infty}$ lying in $L$. Then $f_{n}^{-1}\left(\gamma_{\infty}\right)$ splits into the disjoint union of $n_{0}$ simple loops $\bar{\gamma}_{\infty, i}, i=1, \ldots, n_{0}$. Since $R_{\infty}$ 
is irreducible, each two loops $\bar{\gamma}_{\infty, i}$ and $\bar{\gamma}_{\infty, j}$ belong to the same homology class in $H_{1}\left(X_{n}^{\prime}\right)$ (denote it by $\bar{\gamma}_{\infty}$ ). Therefore $n_{0} \bar{\gamma}_{\infty} \in H_{1}\left(X_{n}^{\prime}\right)_{1}$. Now lemma follows from the remark that $\bar{\gamma}_{1}, \ldots, \bar{\gamma}_{k}, \bar{\gamma}_{\infty}$ generate ker $i_{1}$ and $\bar{\gamma}_{1}, \ldots, \bar{\gamma}_{k}$ generate $H_{1}\left(X_{n}^{\prime}\right)_{1}$.

Lemma 6.4. The homomorphism $i_{2 *}: H_{1}\left(X_{n}, \mathbb{C}\right) \rightarrow H_{1}\left(\bar{X}_{n}, \mathbb{C}\right)$ is an isomorphism.

Proof. We have $X_{n}=\bar{X}_{n} \backslash E$. Denote by $T \subset \bar{X}_{n}$ a closed regular neighbourhood of $E$ and let $\partial T$ be its boundary, $T^{\prime}=T \backslash E$, and $T^{0}=T \backslash \partial T$. It is known (see, for example, the proof of Proposition 3.4 from [5]) that the homomorphism $i_{*}: H_{1}(\partial T, \mathbb{C}) \rightarrow H_{1}(T, \mathbb{C})$, induced by the imbedding $i: \partial T \hookrightarrow T$, is an isomorphism and, besides, there is a deformation retract $T^{\prime} \searrow \partial T$. Therefore there is a deformation retract $X_{n} \searrow X_{n}^{0}$, where $X_{n}^{0}=X_{n} \backslash T^{0}$. Now the lemma follows from the Mayer - Vietories sequence

$$
H_{2}\left(\bar{X}_{n}\right) \rightarrow H_{1}(\partial T) \rightarrow H_{1}(T) \oplus H_{1}\left(X_{n}^{0}\right) \rightarrow H_{1}\left(\bar{X}_{n}\right) \rightarrow 0 .
$$

The proof of Theorem 0.4 follows from Lemmas 6.3, 6.4 and Proposition 6.1

Proposition 6.5. For any $k \in \mathbb{N}$, there exists

(i) an irreducible Hurwitz curve $\bar{H}_{k}$ such that a resolution of singularities $\bar{X}_{k, 6}$ of the cyclic covering of $\mathbb{C P}^{2}$ of degree six, branched along $\bar{H}_{k}$, has first Betti number $b_{1}\left(\bar{X}_{k, 6}\right)=2 k$;

(ii) a Hurwitz curve $\bar{H}_{k}$ consisting of two irreducible components such that the first Betti number $b_{1}\left(\bar{X}_{k, 6}\right)$ of a resolution of singularities $\bar{X}_{k, 6}$ of the cyclic covering of degree six, branched along $\bar{H}$, is equal to $k$.

Proof. In the proof of Proposition 5.18, it was shown that the Alexander polynomial $\Delta(t)$ of a Hurwitz product $G_{2,3}(k)=G_{2,3}^{\diamond k}$ is equal to $\left(t^{2}-\right.$ $t+1)^{k}$ and that the Alexander polynomials $\Delta(t)$ of Hurwitz products $G_{2,3}(2, n)=G(2) \diamond G_{2,3}^{\diamond n}$ and $G_{2,3}(\mathrm{ab}, n)=\mathbb{Z}^{2} \diamond G_{2,3}^{\diamond n}$ respectively are equal to $(t-1)(t+1)\left(t^{2}-t+1\right)^{n}$ and $(1-t)\left(t^{2}-t+1\right)^{n}$.

The groups $G_{2,3}(k), G_{2,3}(2, n)$, and $G_{2,3}(\mathrm{ab}, n)$ are Hurwitz $C$-groups. Moreover, one can assume that the degrees of these Hurwitz $C$-groups are divisible by six (one can take $y^{6}$, where $y$ is the product of the $C$ generators of a Hurwitz $C$-presentation of a group, and apply Lemma 5.11). Therefore by Theorem 6.2 from [12, each of these groups can be realized as the fundamental group $\pi_{1}\left(\mathbb{C}^{2} \backslash H\right)$ for some Hurwitz curve of degree divisible by six. The curve $\bar{H}$ is irreducible in the case of 
$G_{2,3}(k)$ and consists of two irreducible components in the other two cases. Now Theorem 0.4 implies Proposition 6.5.

Proposition 6.6. For any $k \in \mathbb{N}$, there is a Hurwitz curve $\bar{H}_{k}$ which consists of $k+1$ irreducible components, has singularities of the form $w^{2^{3 k-1}}-z^{2^{3 k-1}}=0$, and which is the branch curve of a 2-sheeted cyclic covering $\bar{f}_{2}: \bar{X}_{k, 2} \rightarrow \mathbb{C P}^{2}$ with $b_{1}\left(\bar{X}_{k, 2}\right)=k$.

In particular, the Hurwitz curve $\bar{H}_{1}$ has $\operatorname{deg} \bar{H}_{1}=2^{10}$, the number of singular points of $\bar{H}_{1}$ is equal to $2^{16}$, and all its singular points are of the form $w^{4}-z^{4}=0$.

Proof. A Hurwitz product $G(2, k)=G(2)^{\diamond k}$ is a Hurwitz $C$-group of degree $m=2^{3 k-1}$. By Propositions 5.12 and 5.16, its Alexander polynomial is

$$
\Delta_{k}(t)=(t-1)^{k}(t+1)^{k} .
$$

By Theorem 6.2 from [12], each group $G(2, k)$ can be realized as the fundamental group $\pi_{1}\left(\mathbb{C}^{2} \backslash H_{k}\right)$ for some Hurwitz curve $\bar{H}_{k}$ of even degree and having singularities of the form $w^{m}-z^{m}=0$. Since the multiplicity of the root $t=1$ of the Alexander polynomial $\Delta_{k}(t)$ is equal to $k$, it follows from Lemma 5.7 and Theorem 5.9 that the curve $\bar{H}_{k}$ consists of $k+1$ irreducible components.

By Theorem [0.4 the first Betti number $b_{1}\left(\bar{X}_{k, 2}\right)=k$, since the multiplicity of the root $t=-1$ of the Alexander polynomial $\Delta_{k}(t)$ is equal to $k$.

To prove the existence of a Hurwitz curve $\bar{H}_{1}$ with the desired properties, we should find some integer $m$ and a braid monodromy factorization

$$
\Delta_{m}^{2}=b_{1} \cdot \ldots \cdot b_{n}
$$

of a Hurwitz curve $\bar{H}$ such that the group given by the presentation

$$
<x_{m}, \ldots, x_{m} \mid x_{i}=b_{j}\left(x_{i}\right) \text { for } i=1, \ldots, m, j=1, \ldots, n>
$$

is $C$-isomorphic to $G(2)$.

To find such presentation, let us recall briefly the proof of Theorem 6.2 from [12 and apply it to calculate the invariants of a Hurwitz curve $\bar{H}_{1}$ for which $\pi_{1}\left(\mathbb{C} \backslash H_{1}\right) \simeq G(2)$.

The group $G(2)$ is given by presentation (401). Consider the group

$$
G_{4,4} \simeq<x_{1}, \ldots, x_{4} \mid x_{i}=\Delta_{4}^{2}\left(x_{i}\right) \text { for } i=1, \ldots, 4>,
$$

where $\Delta_{4}$ is the Garside element in $\mathrm{Br}_{4}$. The braid $\Delta_{4}^{2}$ is the braid monodromy of the singularity given by $w^{4}-z^{4}=0$ and $s_{0}=\Delta_{4}^{2}$ (the factorization with a single factor) is the braid monodromy factorization of four lines in $\mathbb{C P}^{2}$ passing through a fixed point. 
To obtain presentation (40), we should add the relations

$$
x_{2}^{2} x_{1} x_{2}^{-2}=x_{4}, \quad x_{4}^{2} x_{2} x_{4}^{-2}=x_{2}, x_{3}=x_{2}
$$

to presentation (47). For this, using notations and notions from [12], in the beginning one should perform the doubling (see Theorem 3.2 from [12]) of the braid monodromy factorization $s_{0}=\Delta_{4}^{2}$ several times in order to have a possibility to move apart the generators $x_{1}, \ldots, x_{4}$ and to change each relation from (48) by the relations $x_{i}=x_{i+4}$ and relations of the form $x_{i}=b\left(x_{i}\right)$, where $b$ is a braid conjugated to the standard generator of a braid group. It is easy to see that in our case it suffices to perform the doubling two times and we obtain the braid monodromy factorization $s_{1}=d^{2}\left(s_{0}\right)$ of $\Delta_{16}^{2}$ (the doubling $d^{2}\left(s_{0}\right)$ is defined in [12] by formula $\left.(25)\right)$, each factor of which is either conjugated to $\Delta_{4}^{2}$ or conjugated to a standard generator of $\mathrm{Br}_{16}$, and the group

$$
<x_{1}, \ldots, x_{16} \mid x_{i}=b\left(x_{i}\right), i=1, \ldots, 16, \text { and } b \text { is a factor of } s_{1}>
$$

is $C$-isomorphic to $G_{4,4}$. The number of factors of $s_{1}$ conjugated to $\Delta_{4}^{2}$ is equal to $4^{2}$.

Then, to add the relations (48) to presentation (47), one can use Lemma 3.4 from [12] three times and obtain a braid monodromy factorization $s_{2}$ of $\Delta_{2^{10}}^{2}$, each factor of which is either conjugated to $\Delta_{4}^{2}$ or conjugated to a standard generator of $\mathrm{Br}_{2^{10}}$. The number of factors of $s_{2}$ conjugated to $\Delta_{4}^{2}$ is equal to $4^{8}$. The factorization $s_{2}$ is a braid monodromy factorization of a Hurwitz curve $\bar{H}_{1}$, deg $\bar{H}_{1}=2^{10}, \bar{H}_{1}$ has $4^{8}$ singular points of the form $w^{4}-z^{4}=0$, and $\pi_{1}\left(\mathbb{C}^{2} \backslash H\right) \cong G(2)$ by construction of $s_{2}$.

\section{REFERENCES}

[1] Auroux D.: Symplectic 4-manifolds as branched coverings of $\mathbb{P}^{2}$. Invent. Math. 139 (2000), 551-602.

[2] Auroux D., Katzarkov L.: Branched coverings of $\mathbb{P}^{2}$ and invariants of symplectic 4-manifolds. Invent. Math. 142 (2000), 631-673.

[3] Burde G., Zieschang H.: Knots. Walter de Gruyter, Berlin · New York, (1985).

[4] Crowell R., Fox R.: Knot Theory. Ginn, Boston, 1963.

[5] Dimca A.: Singularities and Topology of Hypersurfaces. SpringerVerlag, 1992.

[6] Esnault E.: Fibre de Milnor d'un cone sur une courbe plane singuliére. Invent. Math. 68 (1982), 477-496.

[7] Kharlamov V., Kulikov Vik.S.: On braid monodromy factorizations. Izv. Math. 67:3 (2003), 499-534. 
[8] Kohno T.: An algebraic computation of the Alexander polynomial of a plane algebraic curve. Proc. Japan Acad. Ser. A Math. Sci. 59 (1983), 94-97.

[9] Kulikov V.S., Kulikov Vik.S.: On the monodromy and mixed Hodge structure on cohomology of the infinite cyclic covering of the complement to a plane algebraic curve. Izv. Math. 59:2 (1995), 367-386.

[10] Kulikov Vik. S.: Alexander polynomials of plane algebraic curves. Izv. Math. 42:1 (1994), 67-90.

[11] Kulikov Vik. S.: Geometric realization of C-groups. Izv. Math. 45:1 (1995), 197-206.

[12] Kulikov Vik.S.: Full twist factorization formula for double number of strings. Izv. Math., 68:1 (2004).

[13] Kulikova O.V.: On the fundamental groups of the complements of Hurwitz curves. ArXiv math. SG/0409027, submitted to Izv. Math.

[14] Lê Dũng Tráng: Sur les noeuds algébriques, Compositio Math. 25 (1972), 281-321.

[15] Libgober A.: Alexander polynomials of plane algebraic curves and cyclic multiple planes, Duke Math. J., 1982, v. 49, 833-851.

[16] McDuff D. and Salamon D.: Introduction to Symplectic Topology (second edition). Oxford Math. Monographs, Clarendon Press · Oxford, 1998.

[17] Milnor J.: Infinite cyclic covers. Topology of manifolds, 1968, 115-133.

[18] Moishezon B.: The arithmetic of braids and a statement of Chisini. Contemporary Math. 164 (1994), 151-175.

[19] Rolfsen D.: Knots and Links. Mathematical Lecture Series 7, Publish or Perish, 1976.

[20] Randell R.: Milnor fibres and Alexander polynomials of plane curves. Proc. Symp. Pure Math. 40. Part 2. (Arcata Singularities Conference), AMS, 1983, 415-420.

[21] Stein, K: Analytische Zerlegungen komplexer Räume,. Math. Ann. 132 (1956), 63-93.

[22] Zariski O.: Algebraic surfaces. Springer-Verlag, 1971.

KAISERSLAUTERN UNIVERSITY

E-mail address: greuel@mathematik.uni-kl.de

Steklov Mathematical Institute

E-mail address: kulikov@mi.ras.ru 